\title{
Facile Oxide to Chalcogenide Conversion for Actinides using the Boron-Chalcogen Mixture Method
}

Logan S. Breton, $†$ Vladislav V. Klepov,$\dagger^{*}$ and Hans-Conrad zur Loye*

Department of Chemistry and Biochemistry, University of South Carolina, Columbia, SC, 29208

*Corresponding authors.E-mail: zurloye@mailbox.sc.edu,vladislavklepov@gmail.com 


\section{Table of Contents}

1. $\mathrm{ThO}_{2} \rightarrow \mathrm{ThS}_{2}$ PXRD Pattern $\quad \mathrm{S} 3$

2. $\mathrm{UO}_{2} \rightarrow \mathrm{US}_{2}$ Varying Temperature Reactions PXRD Pattern $\quad \mathrm{S} 4$

3. $\mathrm{ThO}_{2} \rightarrow \mathrm{ThS}_{2}$ Varying Temperature Reactions PXRD Pattern S5

4. U-Se Reaction PXRD S6

5. $\mathrm{CoUO}_{4} \mathrm{PXRD}^{\mathrm{P}}$ Pattern $\quad \mathrm{S} 7$

6. $\mathrm{UCoS}_{3}$ PXRD Pattern $\quad$ S8

7. $\mathrm{UNiS}_{3}$ PXRD Pattern $\quad$ S9

8. $\mathrm{UCoS}_{3}$ Magnetism Plots $\quad \mathrm{S} 10$

9. Phase Pure $L n_{\mathrm{x}} \mathrm{U}_{2} \mathrm{~S}_{5}$ PXRD Patterns $\quad \mathrm{S} 12$

10. $L n_{\mathrm{x}} \mathrm{U}_{2} \mathrm{~S}_{5}$ Magnetism Plots $\quad \mathrm{S} 18$

11. Magnetic Susceptibilities and Effective Moments for $L n_{\mathrm{x}} \mathrm{U}_{2} \mathrm{~S}_{5} \quad \mathrm{~S} 24$

$\begin{array}{ll}\text { 12. Crystallographic Details } & \text { S25 }\end{array}$

13. Elemental Compositions $\quad$ S34

14. SEM Images $\quad$ S35

$\begin{array}{ll}\text { 15. SEM Spectra } & \text { S37 }\end{array}$

16. Tabulated Reagent Ratios and Amounts for the Chalcogenation Reactions S43

17. $L n_{\mathrm{x}} \mathrm{U}_{2} \mathrm{~S}_{5}$ Structure Figure $\quad$ S44

$\begin{array}{ll}\text { 18. BVS Results } & \text { S45 }\end{array}$ 


\section{1. $\mathrm{ThO}_{2} \rightarrow \mathrm{ThS}_{2}$ PXRD Pattern}

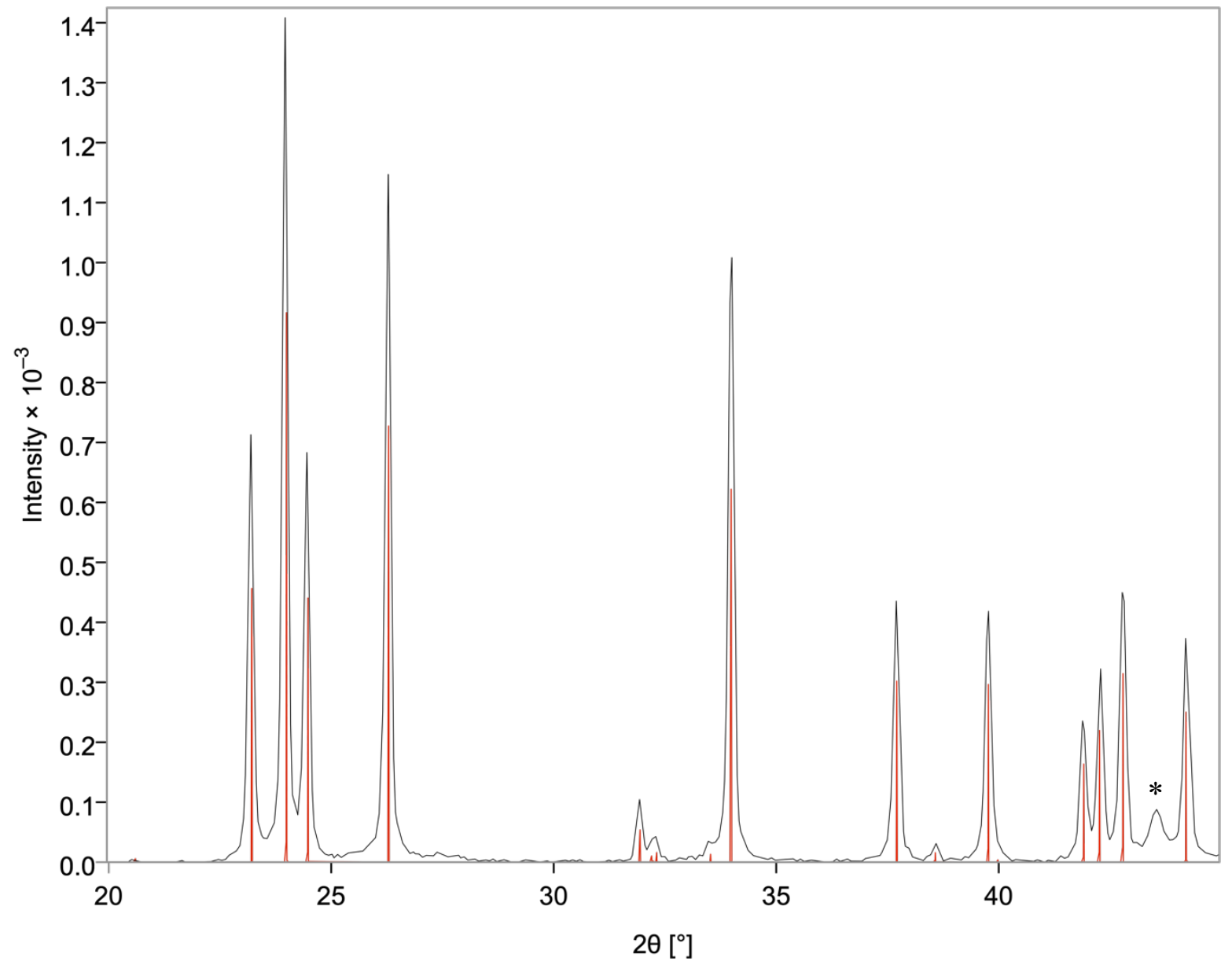

Figure S1. The PXRD of pure $\mathrm{ThS}_{2}$ with the overlaid CIF from the reaction of $\mathrm{ThO}_{2}$ with a mixture of boron and sulfur. Peak at $\sim 43^{\circ}$ (marked with an asterisk) corresponds to the steel slide. 


\section{2. $\mathrm{UO}_{2} \rightarrow \mathrm{US}_{2}$ Varying Temperature Reactions PXRD Pattern}

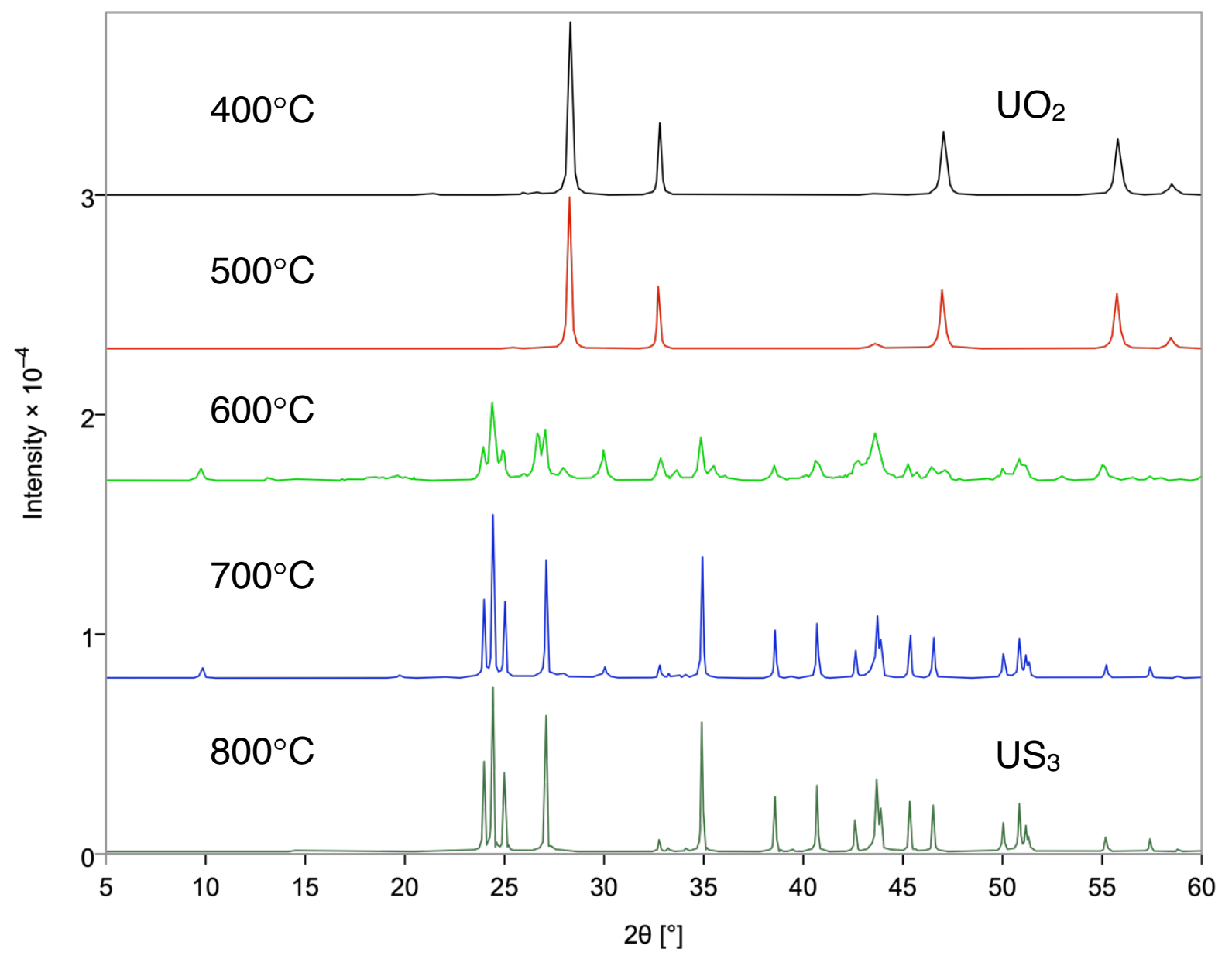

Figure S2. The PXRD patterns of products obtained in a reaction between $\mathrm{U}_{3} \mathrm{O}_{8}, \mathrm{~B}$, and $\mathrm{S}$ performed at (from top to bottom) $400,500,600,700$, and $800{ }^{\circ} \mathrm{C}$. The following products were identified: $\mathrm{UO}_{2}\left(500{ }^{\circ} \mathrm{C}\right) ; \mathrm{UOS}, \mathrm{US}_{3}$, and $\mathrm{US}_{2}\left(600^{\circ} \mathrm{C}\right) ; \mathrm{US}_{3}$ and $\mathrm{US}_{2}\left(700^{\circ} \mathrm{C}\right) ; \mathrm{US}_{2}\left(800^{\circ} \mathrm{C}\right)$. 


\section{3. $\mathrm{ThO}_{2} \rightarrow \mathrm{ThS}_{2}$ Varying Temperature Reactions PXRD Pattern}

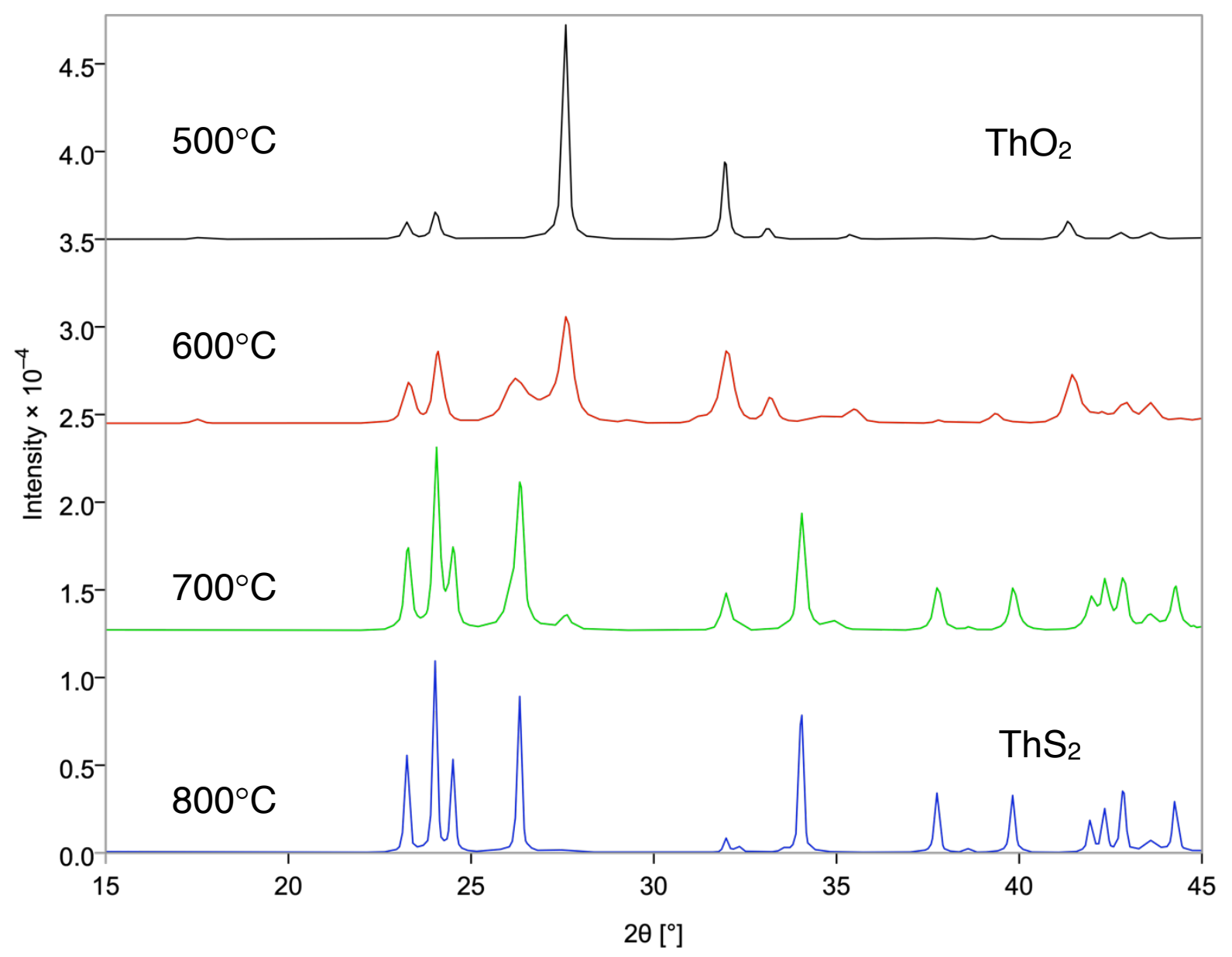

Figure S3. The PXRD patterns of products obtained in a reaction between $\mathrm{ThO}_{2}, \mathrm{~B}$, and $\mathrm{S}$ performed at (from top to bottom) $500,600,700$, and $800{ }^{\circ} \mathrm{C}$. The following products were identified: $\mathrm{ThO}_{2}$ and $\mathrm{Th}_{2} \mathrm{~S}_{5}\left(500{ }^{\circ} \mathrm{C}\right)$; $\mathrm{ThO}_{2}$, ThOS, and $\mathrm{Th}_{2} \mathrm{~S}_{5}\left(600{ }^{\circ} \mathrm{C}\right) ; \mathrm{ThO}_{2}$ and $\mathrm{ThS}_{2}\left(700{ }^{\circ} \mathrm{C}\right)$; $\mathrm{ThS}_{2}\left(800^{\circ} \mathrm{C}\right)$. 


\section{U-Se System PXRD}

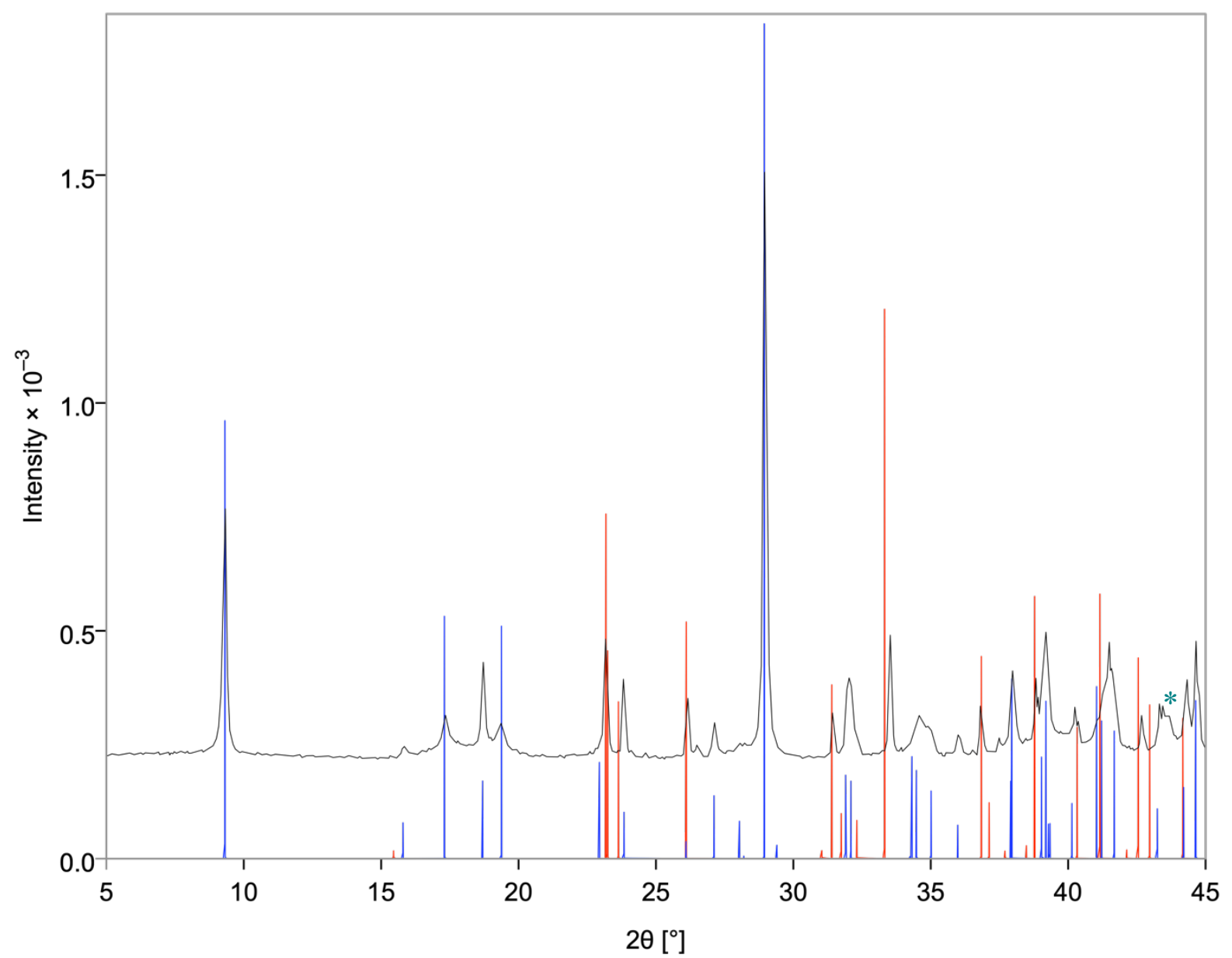

Figure S4. The experimental (black) PXRD pattern of the product of the reaction at $800{ }^{\circ} \mathrm{C}$ between $\mathrm{UO}_{2}, \mathrm{~B}$, and $\mathrm{Se}$ in a 1:2:3 molar ratio, respectively, with the calculated PXRD patterns of the indexed phases, $\mathrm{USe}_{3}$ (blue) and $\mathrm{USe}_{2}$ (red). Peak at $\sim 43^{\circ}$ (marked with an asterisk) corresponds to the steel slide. 


\section{4. $\mathrm{CoUO}_{4} \mathrm{PXRD}$ Pattern}

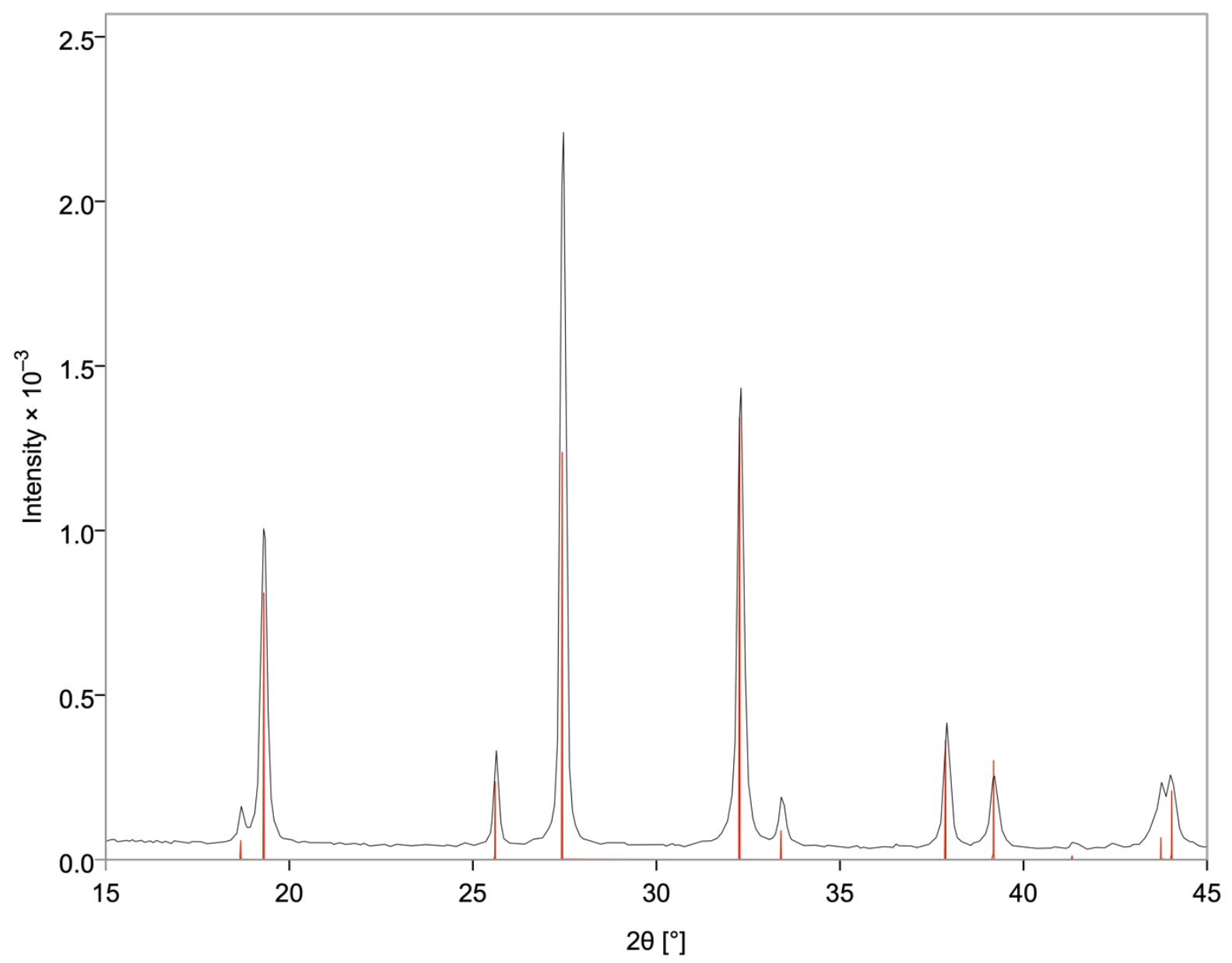

Figure S5. Experimental (black) and calculated (red) PXRD patterns of $\mathrm{CoUO}_{4}$. 


\section{5. $\mathrm{UCoS}_{3}$ PXRD Pattern}

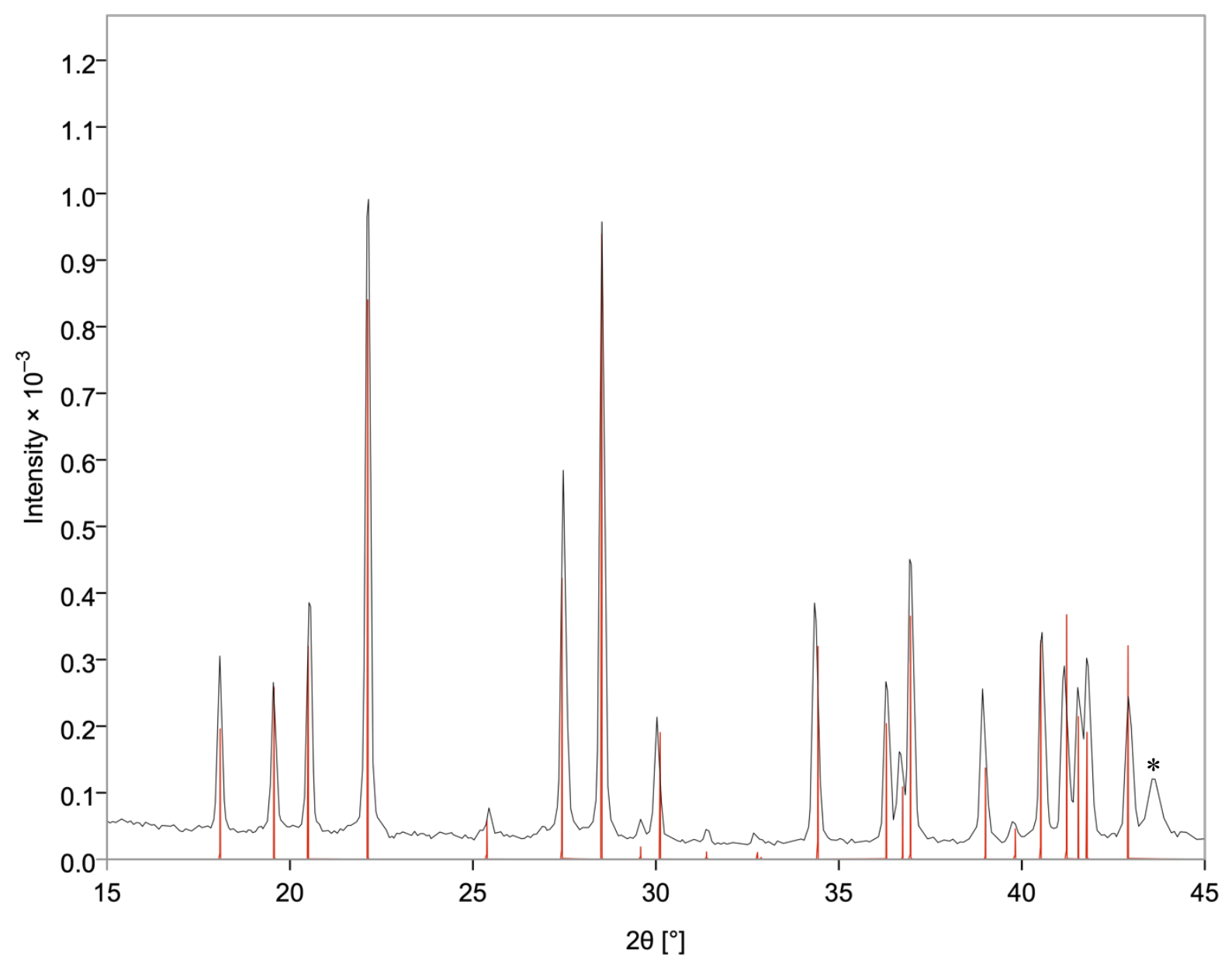

Figure S6. Experimental (black) and calculated (red) PXRD patterns of $\mathrm{UCoS}_{3}$. Peak at $\sim 43^{\circ}$ corresponds to the steel slide. Peak at $\sim 43^{\circ}$ (marked with an asterisk) corresponds to the steel slide. 


\section{6. $\mathrm{UNiS}_{3}$ PXRD Pattern}

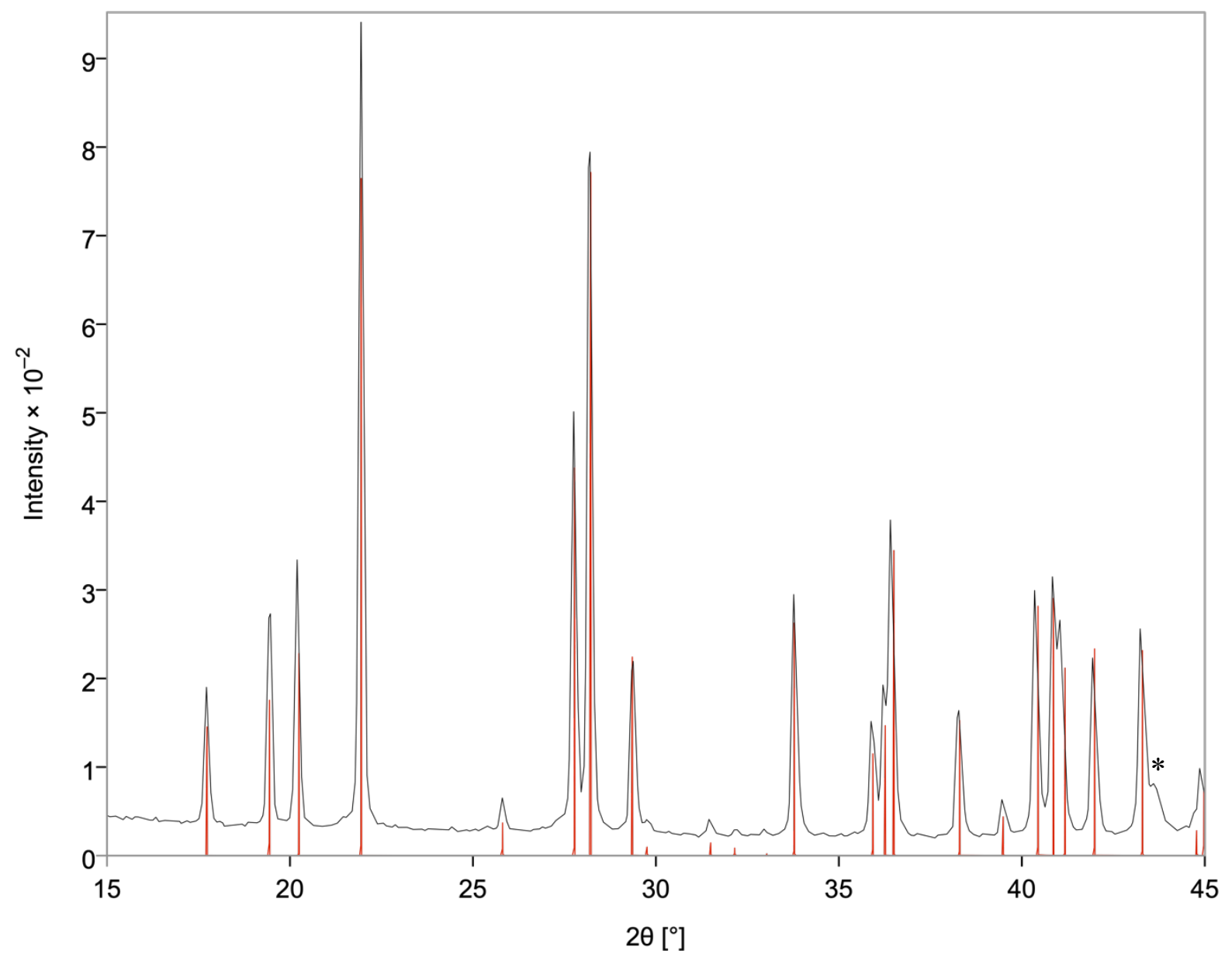

Figure S7. Experimental (black) and calculated (red) PXRD patterns of $\mathrm{UNiS}_{3}$. Peak at $\sim 43^{\circ}$ (marked with an asterisk) corresponds to the steel slide. 
7. $\mathrm{UCoS}_{3}$ Magnetism Plot

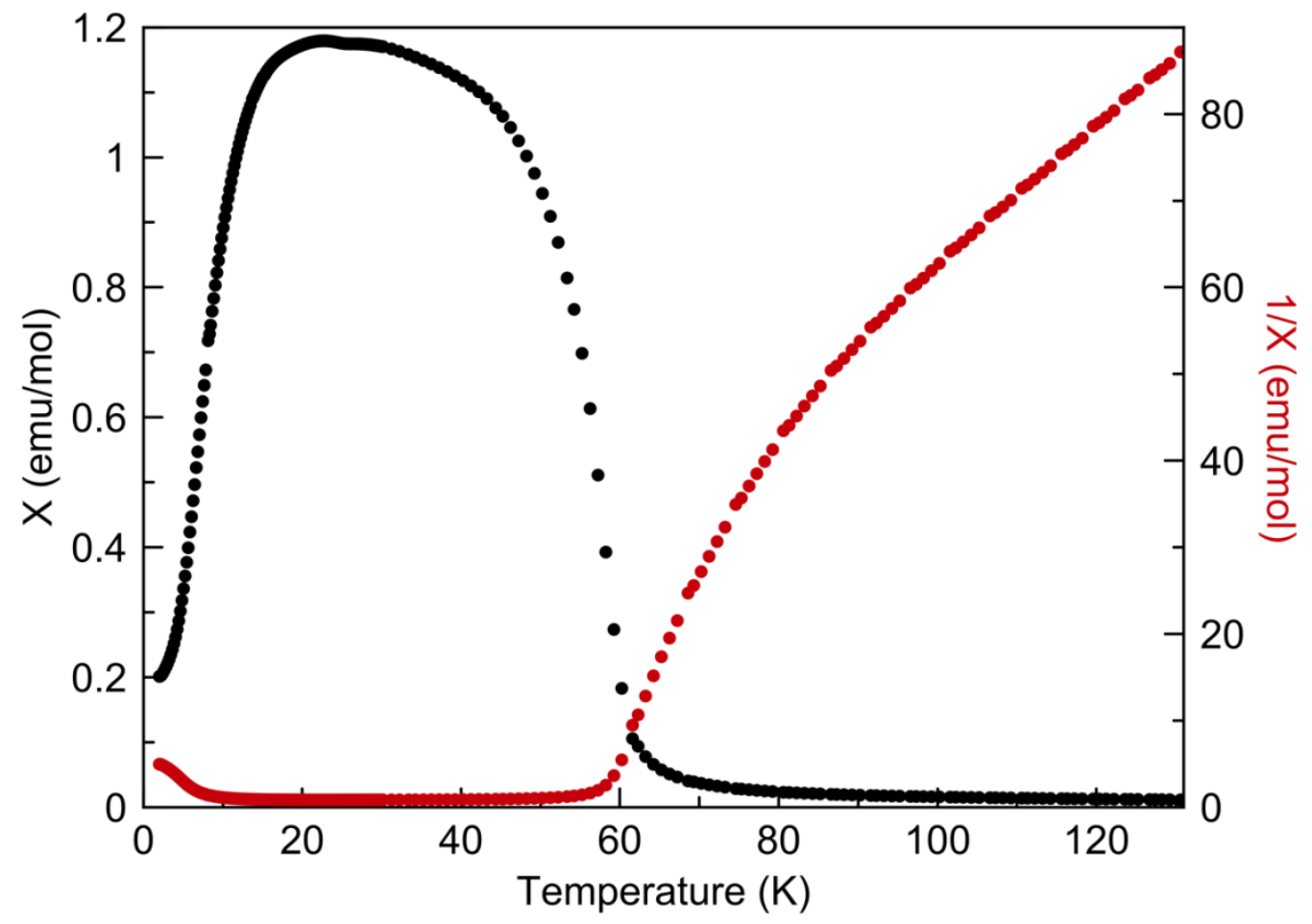

Figure S8. The temperature dependence of the molar susceptibility and inverse molar susceptibility for $\mathrm{UCoS}_{3}$. 


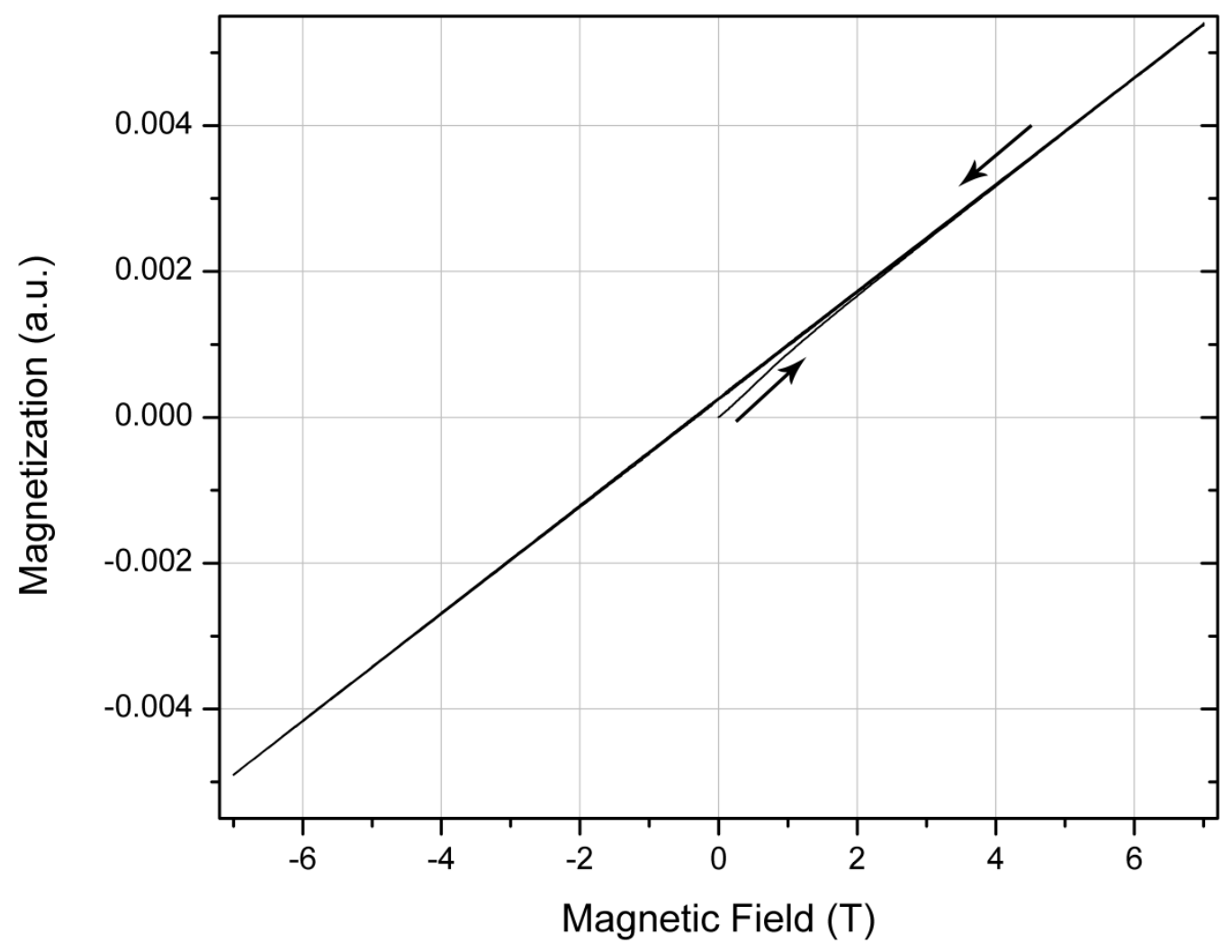

Figure S9. Magnetization vs. field plot for a fresh $\mathrm{UNiS}_{3}$ sample at $300 \mathrm{~K}$. 


\section{Phase Pure $L n_{\mathrm{x}} \mathrm{U}_{2} \mathrm{~S}_{5}$ PXRD Patterns}

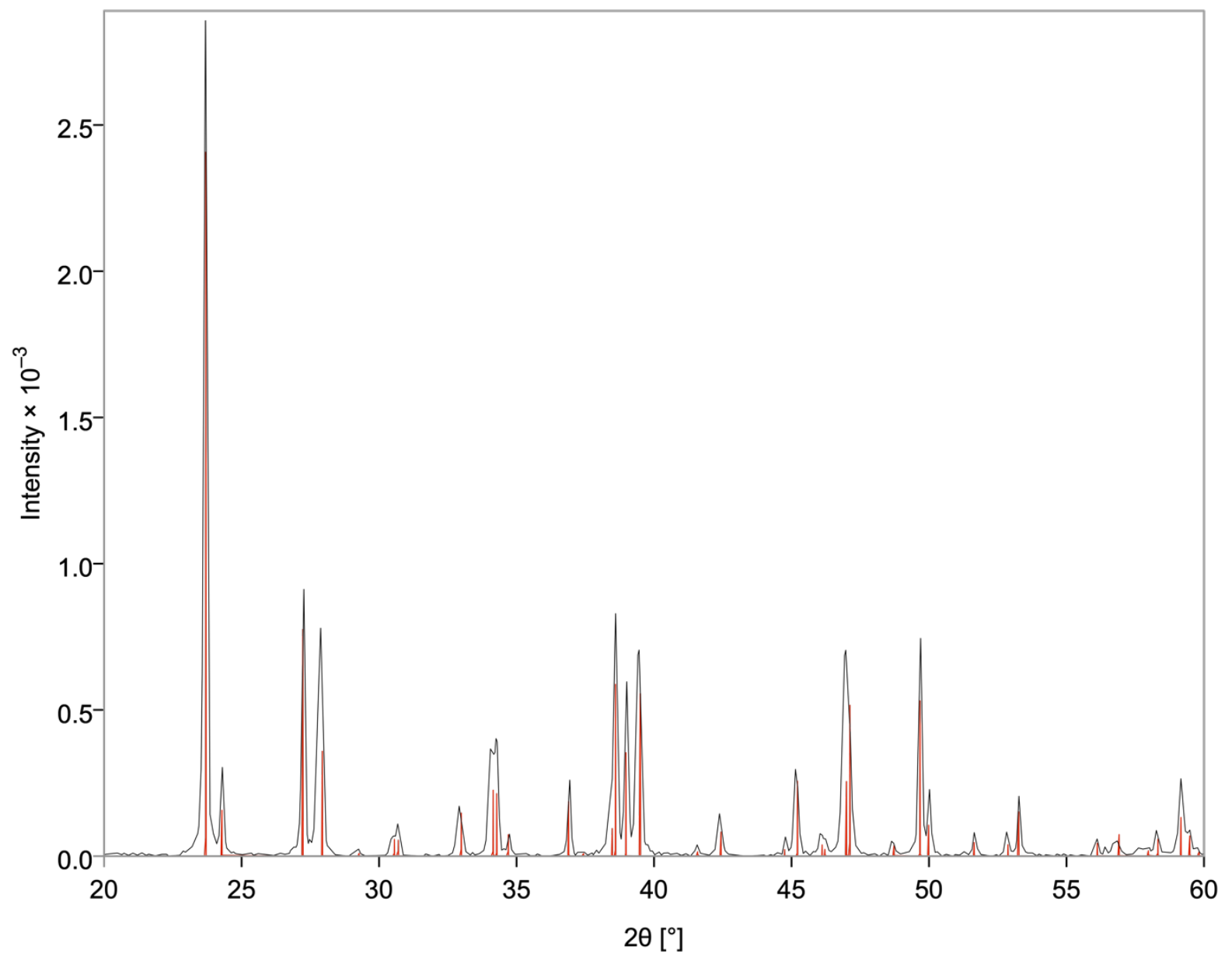

Figure S10. Experimental (black) and calculated (red) PXRD patterns of $\mathrm{Tb}_{0.82(3)} \mathrm{U}_{2} \mathrm{~S}_{5}$. 


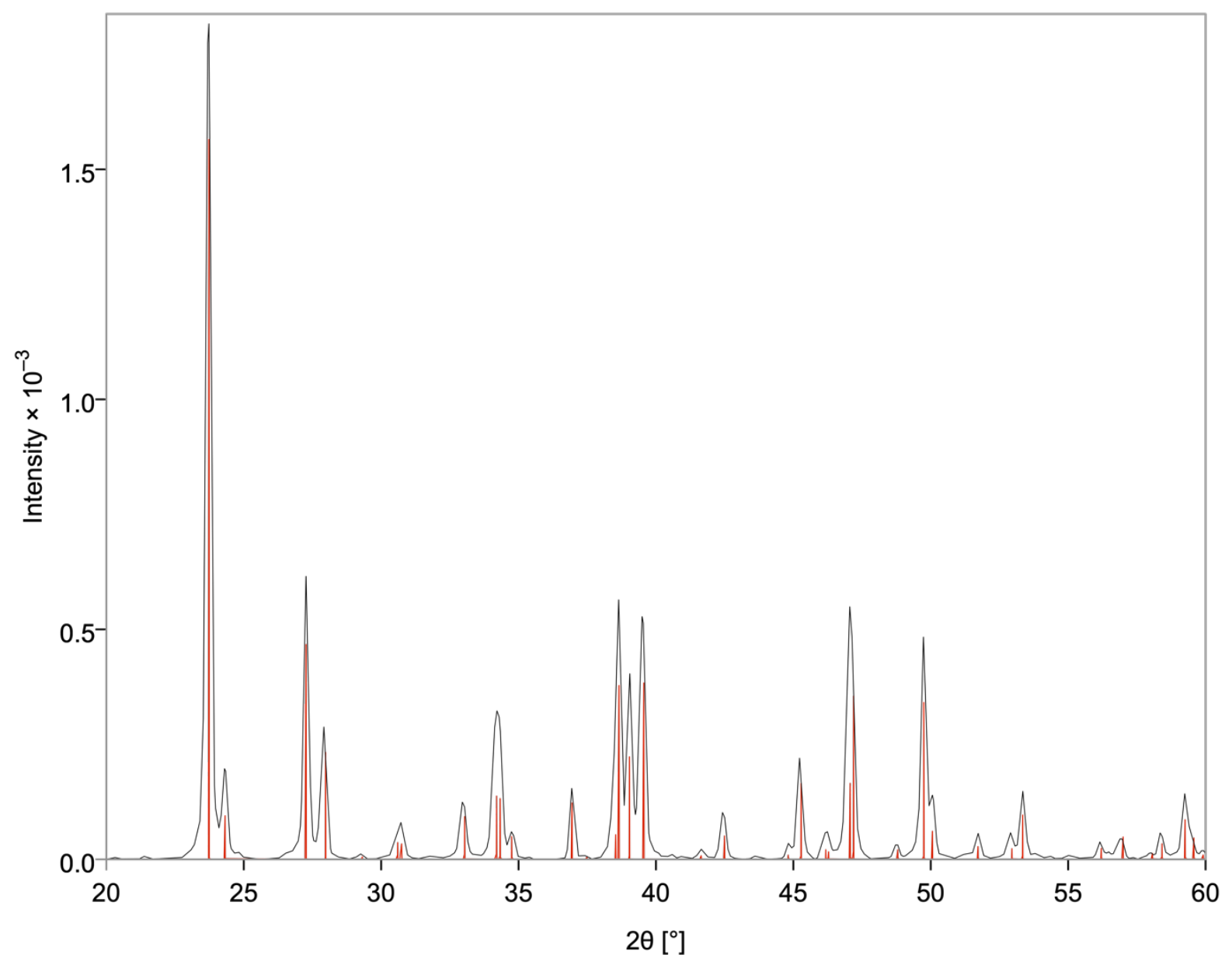

Figure S11. Experimental (black) and calculated (red) PXRD patterns of $\mathrm{Dy}_{0.76(3)} \mathrm{U}_{2} \mathrm{~S}_{5}$. 


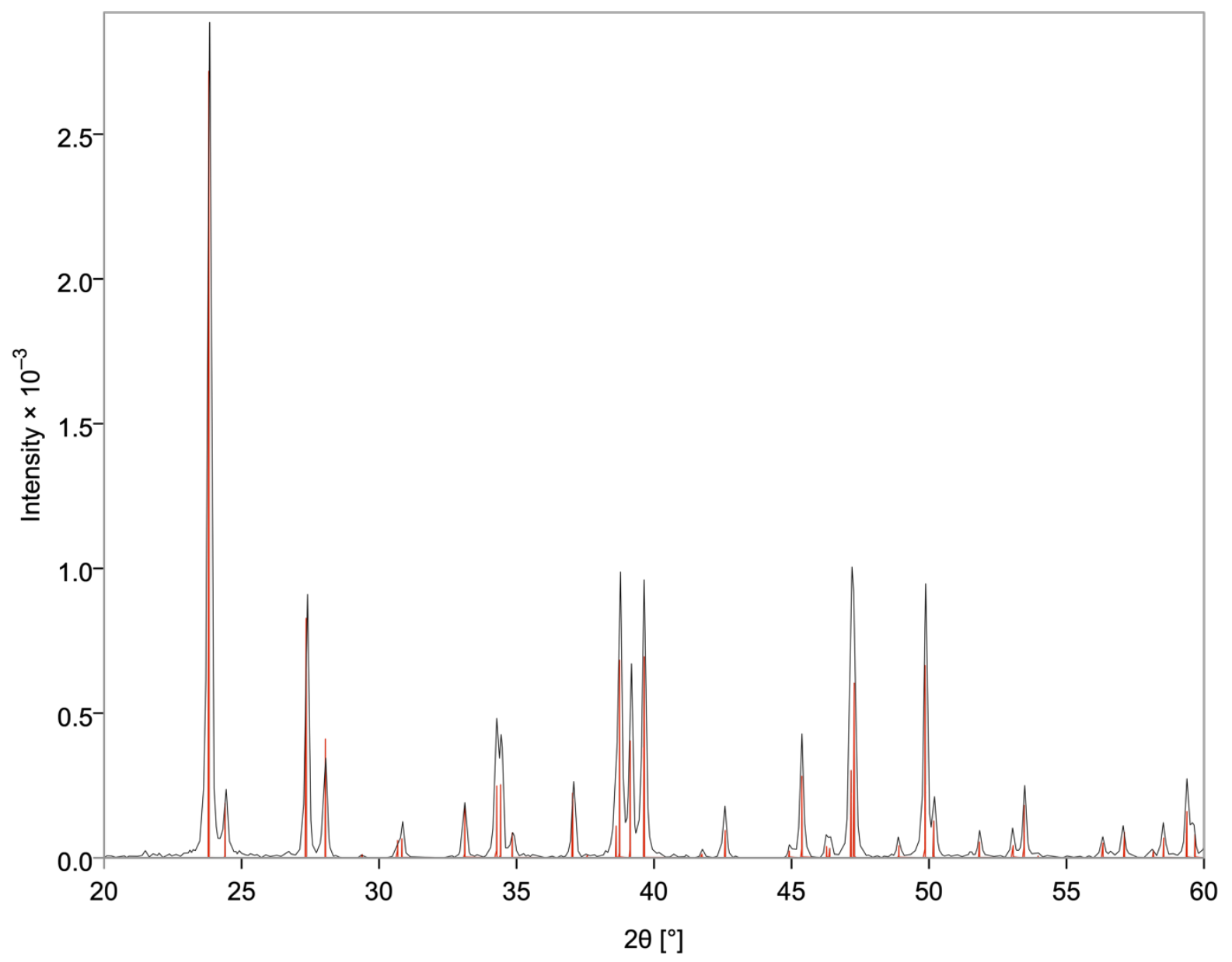

Figure S12. Experimental (black) and calculated (red) PXRD patterns of $\mathrm{Ho}_{0.79(3)} \mathrm{U}_{2} \mathrm{~S}_{5}$. 


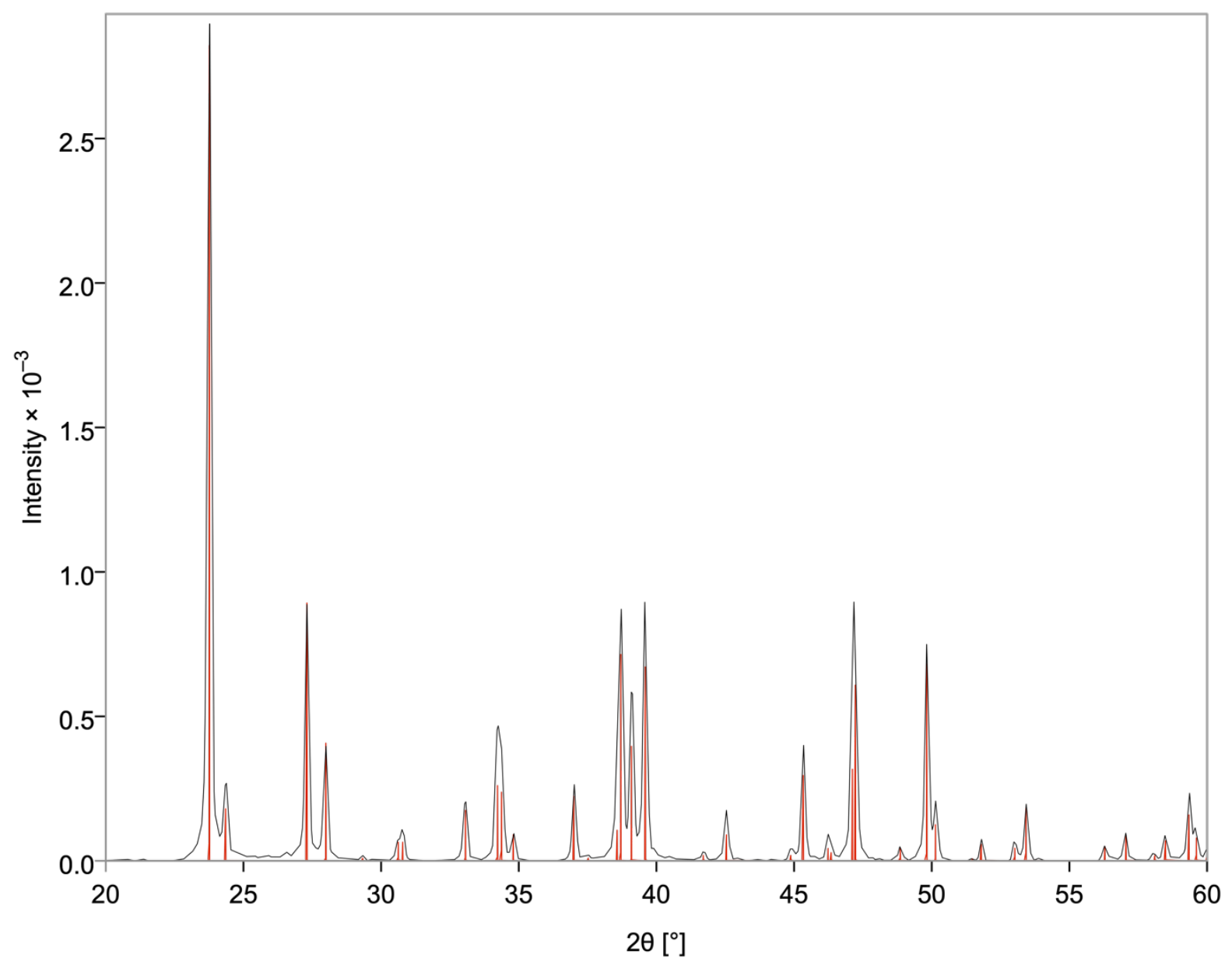

Figure S13. Experimental (black) and calculated (red) PXRD patterns of $\operatorname{Er}_{0.75(1)} \mathrm{U}_{2} \mathrm{~S}_{5}$. 


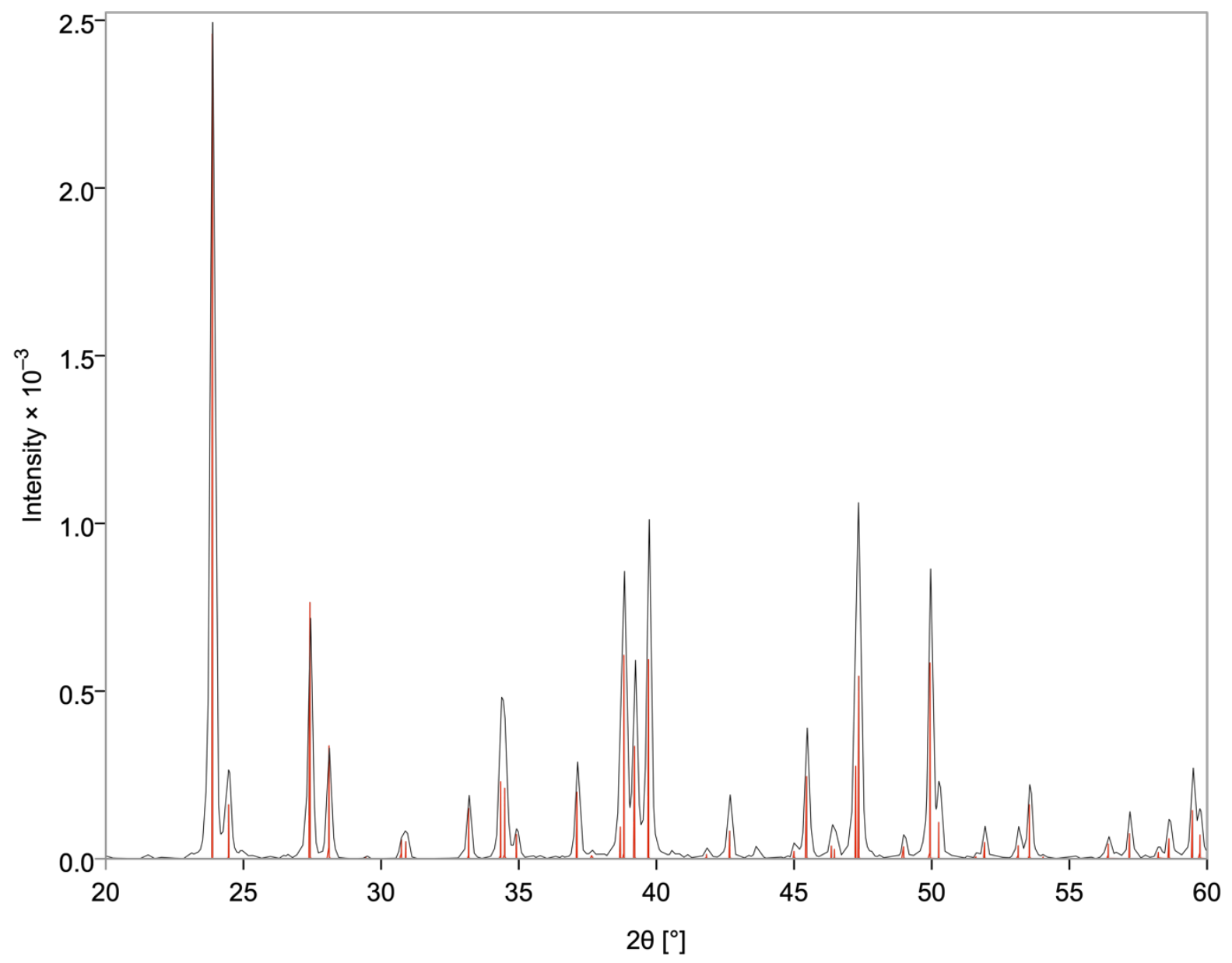

Figure S14. Experimental (black) and calculated (red) PXRD patterns of $\mathrm{Tm}_{0.75(1)} \mathrm{U}_{2} \mathrm{~S}_{5}$. 


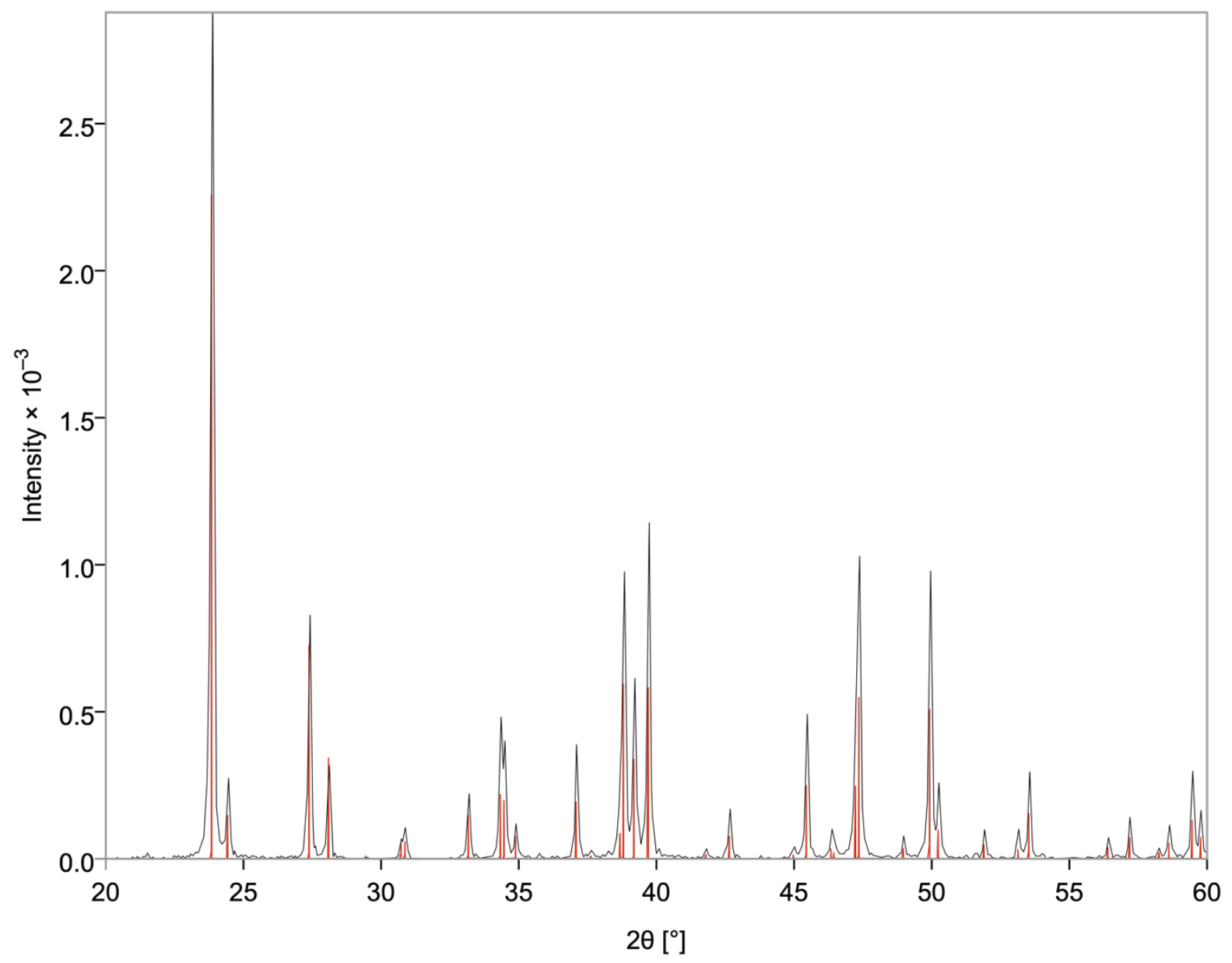

Figure S15. Experimental (black) and calculated (red) PXRD patterns of $\mathrm{Yb}_{0.70(4)} \mathrm{U}_{2} \mathrm{~S}_{5}$. 
9. $L n_{\mathrm{x}} \mathrm{U}_{2} \mathrm{~S}_{5}$ Magnetism Plots

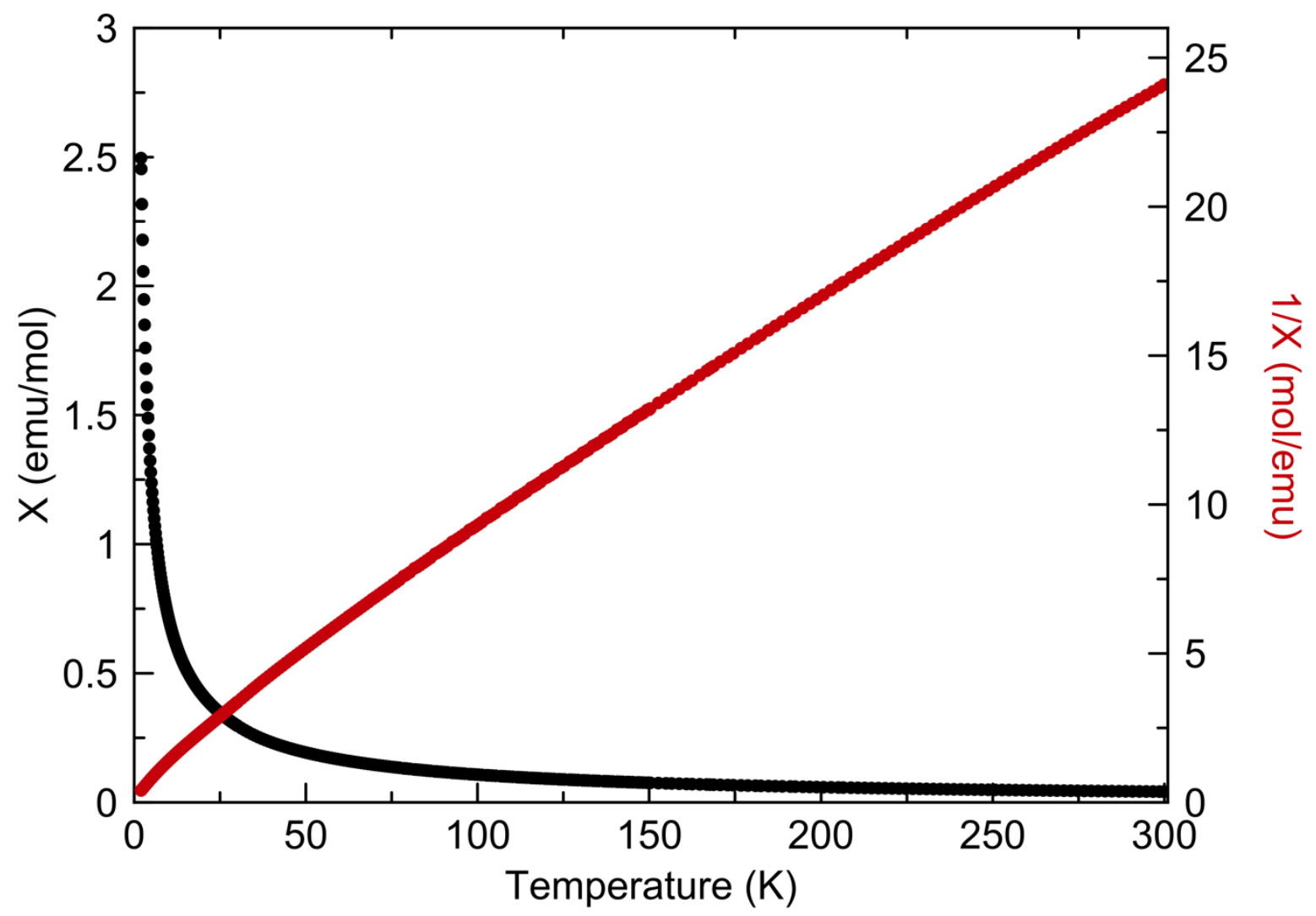

Figure S16. The temperature dependence of the molar susceptibility and inverse molar susceptibility for $T b_{0.82(3)} U_{2} S_{5}$. 


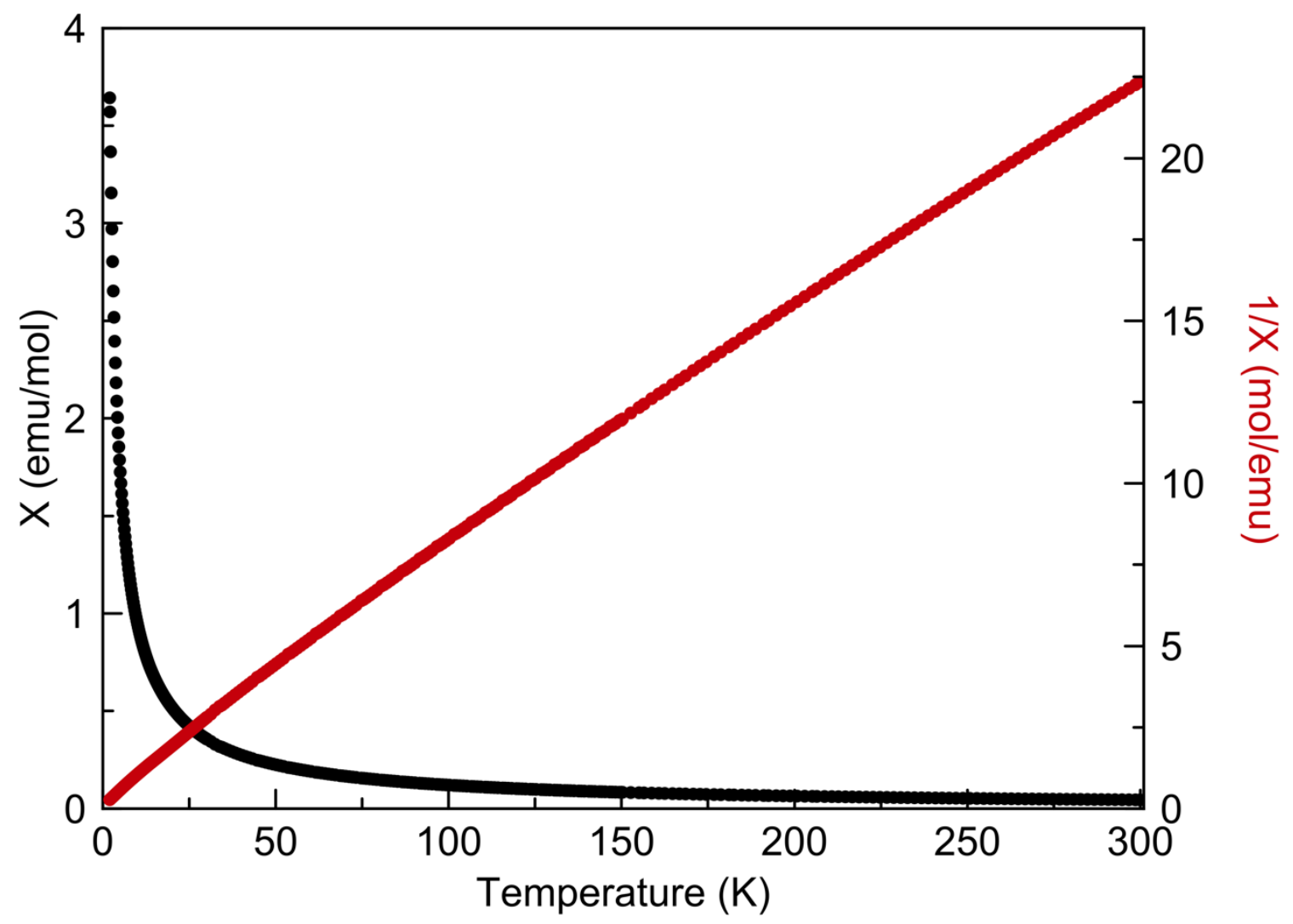

Figure S17. The temperature dependence of the molar susceptibility and inverse molar susceptibility for $\mathrm{Dy}_{0.76(3)} \mathrm{U}_{2} \mathrm{~S}_{5}$. 


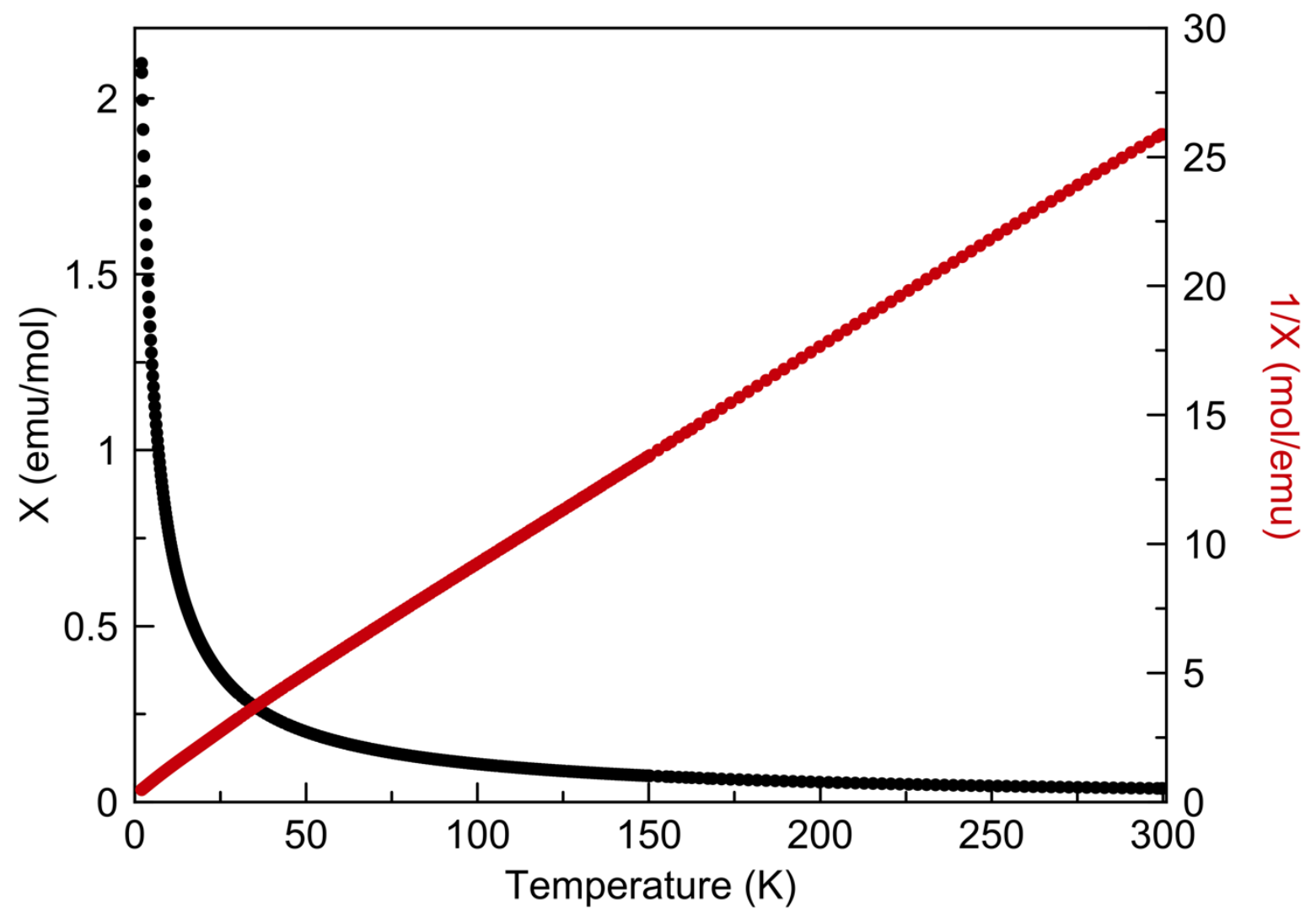

Figure S18. The temperature dependence of the molar susceptibility and inverse molar susceptibility for $\mathrm{Ho}_{0.78(3)} \mathrm{U}_{2} \mathrm{~S}_{5}$. 


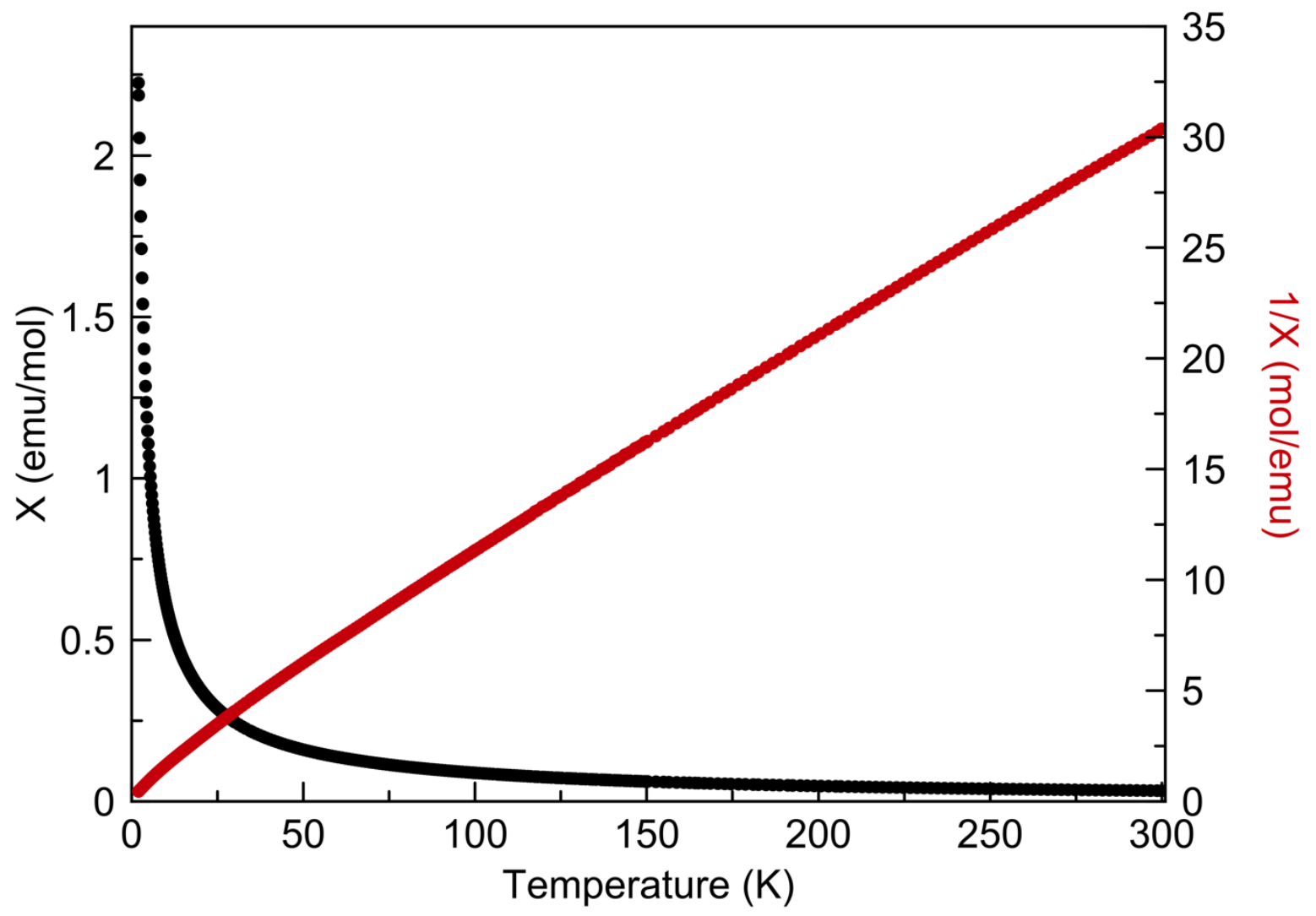

Figure S19. The temperature dependence of the molar susceptibility and inverse molar susceptibility for $\operatorname{Er}_{0.75(1)} \mathrm{U}_{2} \mathrm{~S}_{5}$. 


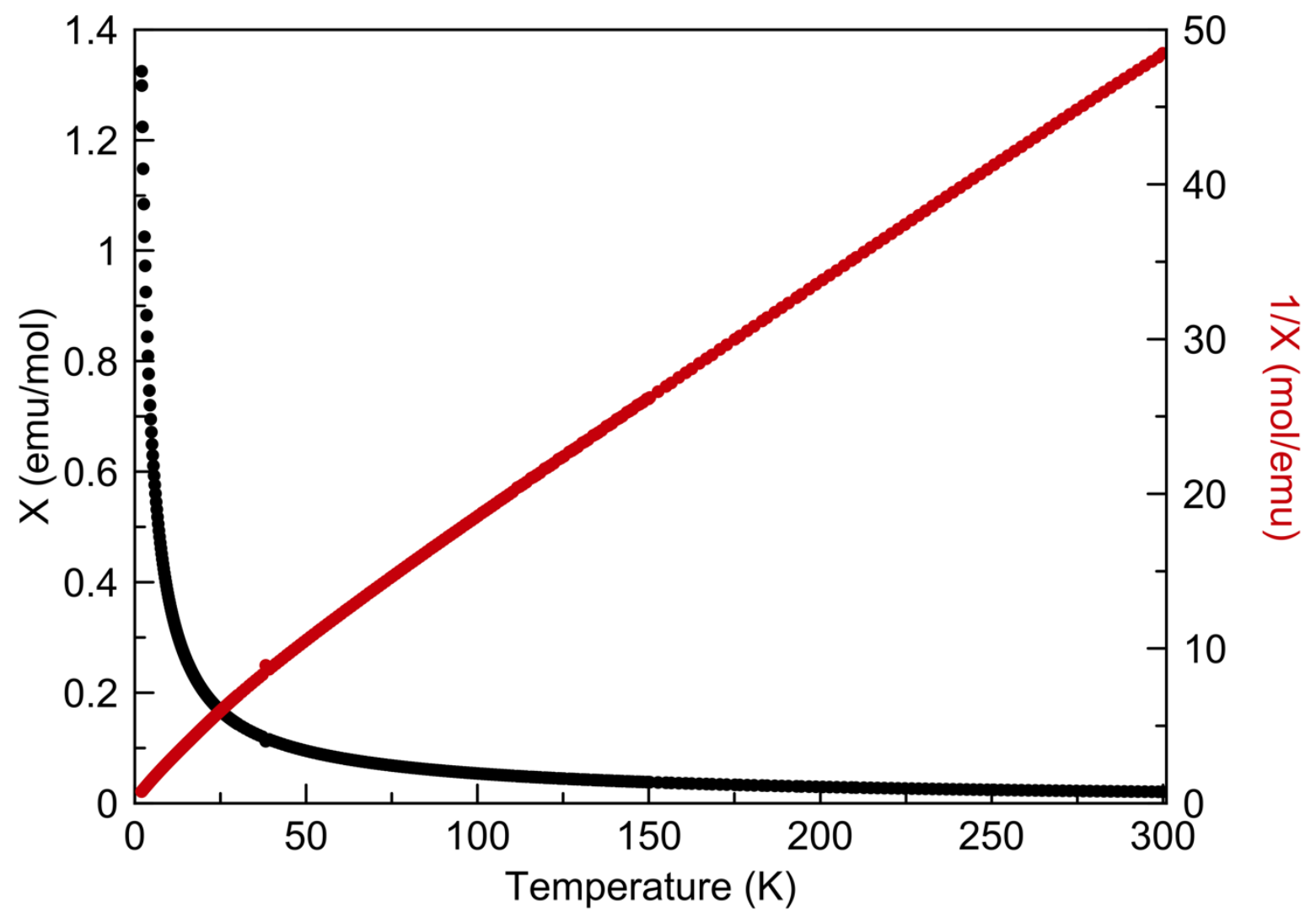

Figure S20. The temperature dependence of the molar susceptibility and inverse molar susceptibility for $\mathrm{Tm}_{0.75(1)} \mathrm{U}_{2} \mathrm{~S}_{5}$. 


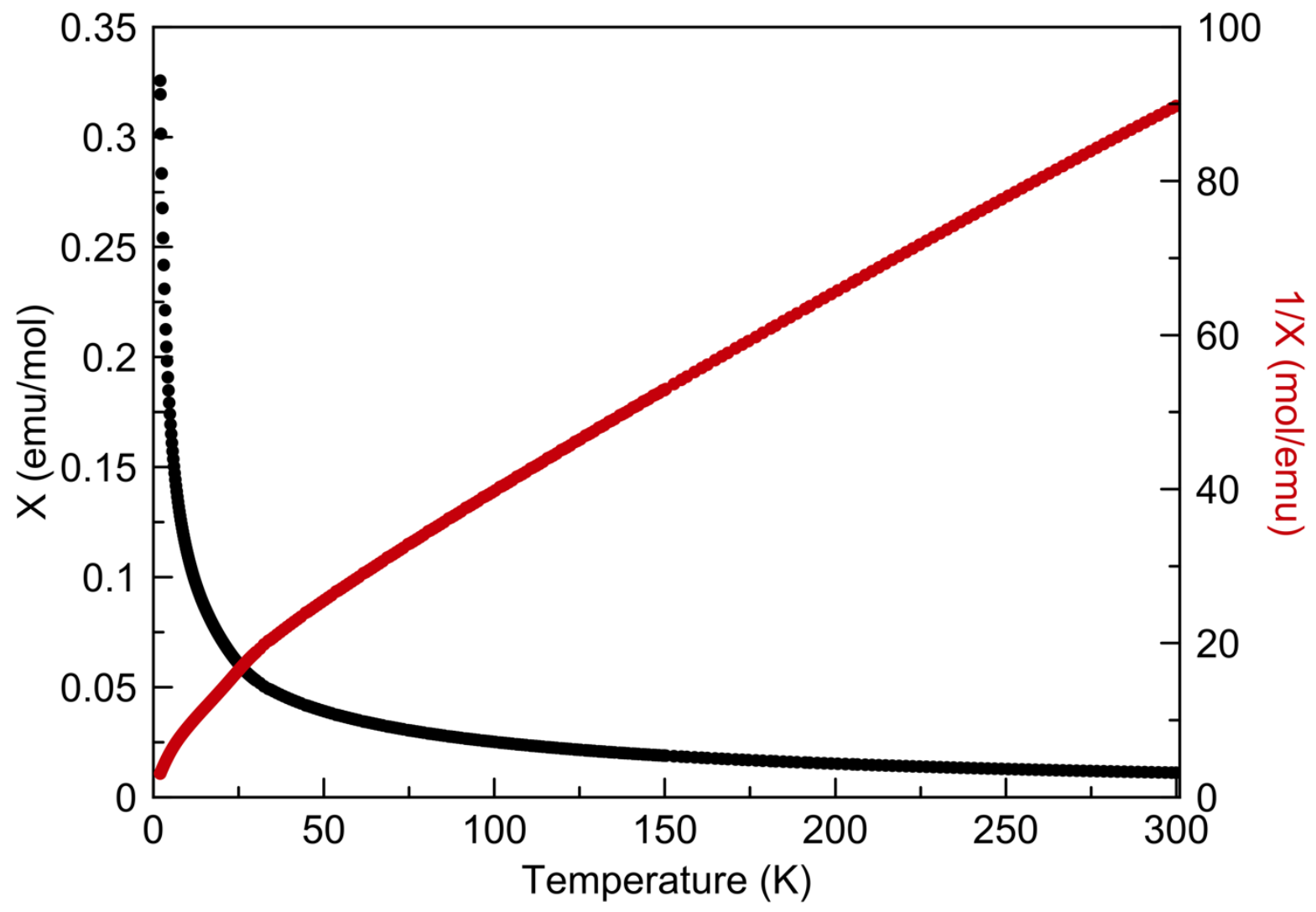

Figure S21. The temperature dependence of the molar susceptibility and inverse molar susceptibility for $\mathrm{Yb}_{0.70(4)} \mathrm{U}_{2} \mathrm{~S}_{5}$. 
10. Magnetic Susceptibilities and Effective Moments for $L n_{x} U_{2} S_{5}$

Table S1. The molar susceptibility, $\chi$, and total effective moment, $\mu_{\mathrm{eff}}$, at $300 \mathrm{~K}$ obtained for phase pure samples of $L n_{\mathrm{x}} \mathrm{U}_{2} \mathrm{~S}_{5}(\mathrm{Ln}=\mathrm{Tb}, \mathrm{Dy}, \mathrm{Ho}, \mathrm{Er}, \mathrm{Tm}, \mathrm{Yb})$.

\begin{tabular}{|c|c|c|}
\hline $\operatorname{Ln}_{\mathrm{x}} \mathrm{U}_{2} \mathrm{~S}_{5}$ & $\chi(\mathrm{emu} / \mathrm{mol})$ & $\mu_{\text {eff }}\left(\mu_{\mathrm{B}}\right)$ \\
\hline $\mathrm{Tb}_{0.82(3)} \mathrm{U}_{2} \mathrm{~S}_{5}$ & 0.0415 & 9.9748 \\
\hline $\mathrm{Dy}_{0.76(3)} \mathrm{U}_{2} \mathrm{~S}_{5}$ & 0.0447 & 10.3567 \\
\hline $\mathrm{Ho}_{0.78(3)} \mathrm{U}_{2} \mathrm{~S}_{5}$ & 0.0387 & 9.6264 \\
\hline $\mathrm{Er}_{0.75(1)} \mathrm{U}_{2} \mathrm{~S}_{5}$ & 0.0329 & 8.8853 \\
\hline $\mathrm{Tm}_{0.75(1)} \mathrm{U}_{2} \mathrm{~S}_{5}$ & 0.0206 & 7.0331 \\
\hline $\mathrm{Yb}_{0.70(4)} \mathrm{U}_{2} \mathrm{~S}_{5}$ & 0.0111 & 5.1689 \\
\hline
\end{tabular}




\section{Crystallographic Details}

Table S2. Crystallographic data for the $L n_{\mathrm{x}} \mathrm{U}_{2} \mathrm{~S}_{5}$ phases with $\mathrm{Ln}=\mathrm{Pr}, \mathrm{Nd}, \mathrm{Sm}, \mathrm{Gd}, \mathrm{Tb}, \mathrm{Dy}$

\begin{tabular}{|c|c|c|c|c|c|}
\hline Chemical formula & $\operatorname{Pr}_{0.89(4)} \mathrm{U}_{2} \mathrm{~S}_{5}$ & $\mathrm{Nd}_{0.88(4)} \mathrm{U}_{2} \mathrm{~S}_{5}$ & $\mathrm{Sm}_{0.87(3)} \mathrm{U}_{2} \mathrm{~S}_{5}$ & $\mathrm{Gd}_{0.88(2)} \mathrm{U}_{2} \mathrm{~S}_{5}$ & $\mathrm{~Tb}_{0.82(3)} \mathrm{U}_{2} \mathrm{~S}_{5}$ \\
\hline Formula weight & 762.12 & 763.29 & 766.79 & 775.53 & 766.67 \\
\hline Crystal system & \multicolumn{5}{|c|}{ Tetragonal } \\
\hline Space group, $Z$ & \multicolumn{5}{|c|}{$P 4 / n c c$} \\
\hline $\mathrm{a}, \AA$ & $10.2982(3)$ & $10.2927(2)$ & $10.2913(2)$ & $10.2902(2)$ & $10.2743(2)$ \\
\hline $\mathrm{b}, \AA$ & $10.2982(3)$ & $10.2927(2)$ & $10.2913(2)$ & $10.2902(2)$ & $10.2743(2)$ \\
\hline $\mathrm{c}, \AA$ & $6.3524(2)$ & $6.3490(2)$ & $6.34450(10)$ & $6.35750(10)$ & $6.3372(2)$ \\
\hline$\beta$, deg. & \multicolumn{5}{|c|}{$90^{\circ}$} \\
\hline $\mathrm{V}, \AA^{3}$ & $673.69(4)$ & 672.61(3) & $671.95(3)$ & 673.18(3) & $668.96(3)$ \\
\hline$\rho_{\text {calcd }}, \mathrm{g} / \mathrm{cm}^{3}$ & 7.514 & 7.538 & 7.580 & 7.652 & 7.612 \\
\hline Radiation $(\lambda, \AA)$ & \multicolumn{5}{|c|}{$\operatorname{Mo~K\alpha }(0.71073 \AA)$} \\
\hline$\mu, \mathrm{mm}^{-1}$ & 55.741 & 56.161 & 56.999 & 58.044 & 58.316 \\
\hline $\mathrm{T}, \mathrm{K}$ & 302.83 & 303.9 & 302.14 & 302.43 & 303.88 \\
\hline Crystal dim., $\mathrm{mm}^{3}$ & $0.06 \times 0.06 \times 0.02$ & $0.06 \times 0.06 \times 0.02$ & $0.06 \times 0.06 \times 0.02$ & $0.06 \times 0.06 \times 0.02$ & $0.06 \times 0.06 \times 0.02$ \\
\hline $2 \theta$ range, deg. & $2.797-32.482$ & $2.799-32.490$ & $2.799-32.499$ & $2.799-32.495$ & $2.804-32.493$ \\
\hline Reflections collected & 11591 & 11311 & 11661 & 12092 & 11368 \\
\hline Data/parameters/restraints & $615 / 26 / 0$ & $612 / 26 / 0$ & $611 / 26 / 0$ & $614 / 26 / 0$ & $608 / 26 / 0$ \\
\hline$R_{\text {int }}$ & 0.0383 & 0.0593 & 0.0420 & 0.0406 & 0.0414 \\
\hline Goodness of fit & 1.164 & 1.153 & 1.139 & 1.117 & 1.204 \\
\hline $\mathrm{R}_{1}(\mathrm{I}>2 \sigma(\mathrm{I}))$ & 0.0162 & 0.0201 & 0.0141 & 0.0143 & 0.0186 \\
\hline $\mathrm{wR}_{2}$ (all data) & 0.0365 & 0.0402 & 0.0351 & 0.0357 & 0.0441 \\
\hline
\end{tabular}


Table S3. Crystallographic data for the $L n_{\mathrm{x}} \mathrm{U}_{2} \mathrm{~S}_{5}$ phases with $\mathrm{Ln}=\mathrm{Dy}, \mathrm{Ho}, \mathrm{Er}, \mathrm{Tm}, \mathrm{Yb}$

\begin{tabular}{|c|c|c|c|c|c|}
\hline Chemical formula & $\mathrm{Dy}_{0.76(3)} \mathrm{U}_{2} \mathrm{~S}_{5}$ & $\mathrm{Ho}_{0.78(3)} \mathrm{U}_{2} \mathrm{~S}_{5}$ & $\mathrm{Er}_{0.75(1)} \mathrm{U}_{2} \mathrm{~S}_{5}$ & $\operatorname{Tm}_{0.75(1)} \mathrm{U}_{2} \mathrm{~S}_{5}$ & $\mathrm{Yb}_{0.70(4)} \mathrm{U}_{2} \mathrm{~S}_{5}$ \\
\hline Formula weight & 760.27 & 765.83 & 762.22 & 762.64 & 757.06 \\
\hline Crystal system & \multicolumn{5}{|c|}{ Tetragonal } \\
\hline Space group, Z & \multicolumn{5}{|c|}{$P 4 / n c c$} \\
\hline $\mathrm{a}, \AA$ & $10.2599(2)$ & $10.2482(5)$ & $10.2459(2)$ & $10.2421(2)$ & $10.2367(2)$ \\
\hline $\mathrm{b}, \AA$ & $10.2599(2)$ & $10.2482(5)$ & $10.2459(2)$ & $10.2421(2)$ & $10.2367(2)$ \\
\hline c, $\AA$ & $6.32830(10)$ & $6.3239(3)$ & $6.32500(10)$ & $6.32210(10)$ & $6.3145(2)$ \\
\hline$\beta$, deg. & \multicolumn{5}{|c|}{$90^{\circ}$} \\
\hline $\mathrm{V}, \AA^{3}$ & 666.15 & $664.17(7)$ & $663.99(3)$ & $663.19(3)$ & $661.70(3)$ \\
\hline$\rho_{\text {calcd }}, \mathrm{g} / \mathrm{cm}^{3}$ & 7.581 & 7.659 & 7.625 & 7.638 & 7.599 \\
\hline Radiation $(\lambda, \AA)$ & \multicolumn{5}{|c|}{$\operatorname{Mo~K\alpha }(0.71073 \AA)$} \\
\hline$\mu, \mathrm{mm}^{-1}$ & 58.419 & 59.363 & 59.541 & 60.091 & 60.071 \\
\hline $\mathrm{T}, \mathrm{K}$ & 301.92 & 303.21 & 302.56 & 302.82 & 302.32 \\
\hline Crystal dim., $\mathrm{mm}^{3}$ & $0.06 \times 0.06 \times 0.02$ & $0.06 \times 0.06 \times 0.02$ & $0.06 \times 0.06 \times 0.02$ & $0.06 \times 0.06 \times 0.02$ & $0.06 \times 0.06 \times 0.02$ \\
\hline $2 \theta$ range, deg. & $2.808-32.499$ & $2.811-29.977$ & $2.811-39.097$ & $2.813-39.112$ & $2.814-29.925$ \\
\hline Reflections collected & 11396 & 7138 & 60859 & 59232 & 38623 \\
\hline Data/parameters/restraints & $606 / 26 / 0$ & $486 / 26 / 0$ & $970 / 26 / 0$ & $968 / 26 / 0$ & $485 / 26 / 0$ \\
\hline$R_{\text {int }}$ & 0.0474 & 0.0505 & 0.0676 & 0.0759 & 0.0647 \\
\hline Goodness of fit & 1.156 & 1.125 & 1.195 & 1.182 & 1.291 \\
\hline $\mathrm{R}_{1}(\mathrm{I}>2 \sigma(\mathrm{I}))$ & 0.0180 & 0.0224 & 0.0152 & 0.0188 & 0.0335 \\
\hline $\mathrm{wR}_{2}$ (all data) & 0.0421 & 0.0566 & 0.0325 & 0.0460 & 0.0919 \\
\hline
\end{tabular}


Table S4. Crystallographic data for $\mathrm{Rb}_{1.72} \mathrm{Na}_{0.68} \mathrm{I}_{0.40}\left[\mathrm{Th}\left(\mathrm{PS}_{4}\right)_{2}\right]$ and $\mathrm{Cs}_{6} \mathrm{Cu}_{12} \mathrm{U}_{2} \mathrm{~S}_{15}$

\begin{tabular}{|c|c|c|}
\hline Chemical formula & $\mathrm{Rb}_{1.72} \mathrm{Na}_{0.68} \mathrm{I}_{0.40}\left[\mathrm{Th}\left(\mathrm{PS}_{4}\right)_{2}\right]$ & $\mathrm{Cs}_{6} \mathrm{Cu}_{12} \mathrm{U}_{2} \mathrm{~S}_{15}$ \\
\hline Formula weight & 763.86 & 2517.00 \\
\hline Crystal system & Orthorhombic & Cubic \\
\hline Space group, $\mathrm{Z}$ & Ccce & $I a-3 d$ \\
\hline $\mathrm{a}, \AA$ & $10.5566(4)$ & $19.0822(4)$ \\
\hline $\mathrm{b}, \AA$ & $20.6415(9)$ & $19.0822(4)$ \\
\hline $\mathrm{c}, \AA$ & $38.2850(15)$ & $19.0822(4)$ \\
\hline$\beta$, deg. & \multicolumn{2}{|c|}{$90^{\circ}$} \\
\hline $\mathrm{V}, \AA^{3}$ & $8342.5(6)$ & $6948.4(4)$ \\
\hline$\rho_{\text {calcd }}, \mathrm{g} / \mathrm{cm}^{3}$ & 3.041 & 4.812 \\
\hline Radiation $(\lambda, \AA)$ & \multicolumn{2}{|c|}{ Mo Ka $(0.71073 \AA)$} \\
\hline$\mu, \mathrm{mm}^{-1}$ & 15.837 & 23.559 \\
\hline $\mathrm{T}, \mathrm{K}$ & 301.69 & 301.36 \\
\hline Crystal dim., $\mathrm{mm}^{3}$ & $0.14 \times 0.02 \times 0.01$ & $0.04 \times 0.03 \times 0.02$ \\
\hline $2 \theta$ range, deg. & $2.231-27.499$ & $2.615-27.481$ \\
\hline $\begin{array}{c}\text { Reflections } \\
\text { collected }\end{array}$ & 227538 & 24038 \\
\hline $\begin{array}{c}\text { Data/parameters/re } \\
\text { straints }\end{array}$ & $4799 / 220 / 13$ & $676 / 29 / 0$ \\
\hline$R_{\text {int }}$ & 0.0612 & 0.0405 \\
\hline Goodness of fit & 1.174 & 1.170 \\
\hline $\mathrm{R}_{1}(\mathrm{I}>2 \sigma(\mathrm{I}))$ & 0.0391 & 0.0245 \\
\hline $\mathrm{wR}_{2}$ (all data) & 0.0875 & 0.0485 \\
\hline
\end{tabular}


Table S5. Fractional Atomic Coordinates $\left(\times 10^{4}\right)$ and Equivalent Isotropic Displacement Parameters $\left(\AA^{2} \times 10^{3}\right)$ for $\operatorname{Pr}_{0.89(4)} \mathrm{U}_{2} \mathrm{~S}_{5}$. $\mathrm{U}_{\text {eq }}$ is defined as $1 / 3$ of the trace of the orthogonalised $\mathrm{U}_{\mathrm{IJ}}$ tensor.

\begin{tabular}{|c|c|c|c|c|}
\hline Atom & $\boldsymbol{x}$ & $\boldsymbol{y}$ & $\boldsymbol{z}$ & $\mathbf{U}(\mathbf{e q})$ \\
\hline $\mathrm{U} 1$ & $5957.4(2)$ & $957.4(2)$ & 7500 & $11.28(8)$ \\
\hline Pr1 & 2500 & 2500 & $1669(16)$ & $8.9(6)$ \\
\hline S1 & 7500 & 2500 & 10000 & $8.3(3)$ \\
\hline S2 & $4589.3(9)$ & $1607.5(9)$ & $3831.4(15)$ & $11.35(18)$ \\
\hline Pr2 & 2500 & 2500 & $1120(20)$ & $12.0(8)$ \\
\hline
\end{tabular}

Table S6. Fractional Atomic Coordinates $\left(\times 10^{4}\right)$ and Equivalent Isotropic Displacement Parameters $\left(\AA^{2} \times 10^{3}\right)$ for $\mathrm{Nd}_{0.88(4)} \mathrm{U}_{2} \mathrm{~S}_{5}$. $\mathrm{U}_{\text {eq }}$ is defined as $1 / 3$ of the trace of the orthogonalised $\mathrm{U}_{\mathrm{IJ}}$ tensor.

\begin{tabular}{|c|c|c|c|c|}
\hline Atom & $\boldsymbol{x}$ & $\boldsymbol{y}$ & $\boldsymbol{z}$ & $\mathbf{U}(\mathbf{e q})$ \\
\hline $\mathrm{U} 1$ & $5958.4(2)$ & $4041.6(2)$ & 7500 & $11.95(9)$ \\
\hline $\mathrm{Nd} 1$ & 2500 & 2500 & $1702(15)$ & $8.9(6)$ \\
\hline $\mathrm{S} 1$ & 7500 & 2500 & 5000 & $8.8(3)$ \\
\hline $\mathrm{S} 1$ & $4590.5(9)$ & $3391.3(8)$ & $3830.1(13)$ & $12.11(17)$ \\
\hline $\mathrm{Nd} 2$ & 2500 & 2500 & $1130(20)$ & $13.6(8)$ \\
\hline
\end{tabular}

Table S7. Fractional Atomic Coordinates $\left(\times 10^{4}\right)$ and Equivalent Isotropic Displacement Parameters $\left(\AA^{2} \times 10^{3}\right)$ for $\mathrm{Sm}_{0.87(3)} \mathrm{U}_{2} \mathrm{~S}_{5}$. $\mathrm{U}_{\text {eq }}$ is defined as $1 / 3$ of the trace of the orthogonalised $\mathrm{U}_{\mathrm{IJ}}$ tensor.

\begin{tabular}{|c|c|c|c|c|}
\hline Atom & $\boldsymbol{x}$ & $\boldsymbol{y}$ & $\boldsymbol{z}$ & $\mathbf{U}(\mathbf{e q})$ \\
\hline $\mathrm{U} 1$ & $5961.0(2)$ & $4039.0(2)$ & 7500 & $11.50(8)$ \\
\hline $\mathrm{Sm} 1$ & 2500 & 2500 & $1716(14)$ & $8.9(6)$ \\
\hline $\mathrm{S} 1$ & 7500 & 2500 & 5000 & $8.1(2)$ \\
\hline $\mathrm{S} 2$ & $4591.3(8)$ & $3393.5(8)$ & $3826.4(12)$ & $11.31(15)$ \\
\hline $\mathrm{Sm} 2$ & 2500 & 2500 & $1061(17)$ & $11.6(7)$ \\
\hline
\end{tabular}


Table S8. Fractional Atomic Coordinates $\left(\times 10^{4}\right)$ and Equivalent Isotropic Displacement Parameters $\left(\AA^{2} \times 10^{3}\right)$ for $\mathrm{Gd}_{0.88(2)} \mathrm{U}_{2} \mathrm{~S}_{5}$. $\mathrm{U}_{\text {eq }}$ is defined as $1 / 3$ of the trace of the orthogonalised $\mathrm{U}_{\mathrm{IJ}}$ tensor.

\begin{tabular}{|c|c|c|c|c|}
\hline Atom & $\boldsymbol{x}$ & $\boldsymbol{y}$ & $\boldsymbol{z}$ & $\mathbf{U}(\mathbf{e q})$ \\
\hline $\mathrm{U} 1$ & $4033.8(2)$ & $9033.8(2)$ & 2500 & $11.29(8)$ \\
\hline $\mathrm{S} 1$ & 2500 & 7500 & 0 & $7.7(2)$ \\
\hline $\mathrm{S} 2$ & $5409.3(8)$ & $8395.0(7)$ & $6175.4(12)$ & $10.81(15)$ \\
\hline $\mathrm{Gd} 1$ & 7500 & 7500 & $8247(12)$ & $8.9(6)$ \\
\hline $\mathrm{Gd} 2$ & 7500 & 7500 & $9003(13)$ & $10.6(6)$ \\
\hline
\end{tabular}

Table S9. Fractional Atomic Coordinates $\left(\times 10^{4}\right)$ and Equivalent Isotropic Displacement Parameters $\left(\AA^{2} \times 10^{3}\right)$ for $\mathrm{Tb}_{0.82(3)} \mathrm{U}_{2} \mathrm{~S}_{5}$. $\mathrm{U}_{\text {eq }}$ is defined as $1 / 3$ of the trace of the orthogonalised $\mathrm{U}_{\mathrm{IJ}}$ tensor.

\begin{tabular}{|c|c|c|c|c|}
\hline Atom & $\boldsymbol{x}$ & $\boldsymbol{y}$ & $\boldsymbol{z}$ & $\mathbf{U}(\mathbf{e q})$ \\
\hline $\mathrm{U} 1$ & $9037.5(2)$ & $4037.5(2)$ & 7500 & $11.03(10)$ \\
\hline $\mathrm{Tb} 1$ & 7500 & 7500 & $1781(15)$ & $6.8(7)$ \\
\hline $\mathrm{S} 1$ & 7500 & 2500 & 5000 & $7.9(3)$ \\
\hline $\mathrm{S} 2$ & $8396.3(9)$ & $5408.2(9)$ & $3829.3(14)$ & $10.60(18)$ \\
\hline $\mathrm{Tb} 1$ & 7500 & 7500 & $1065(17)$ & $11.6(8)$ \\
\hline
\end{tabular}

Table S10. Fractional Atomic Coordinates $\left(\times 10^{4}\right)$ and Equivalent Isotropic Displacement Parameters $\left(\AA^{2} \times 10^{3}\right)$ for $\mathrm{Dy}_{0.76(3)} \mathrm{U}_{2} \mathrm{~S}_{5}$. $\mathrm{U}_{\text {eq }}$ is defined as $1 / 3$ of the trace of the orthogonalised $\mathrm{U}_{\mathrm{IJ}}$ tensor.

\begin{tabular}{|c|c|c|c|c|}
\hline Atom & $\boldsymbol{x}$ & $\boldsymbol{y}$ & $\boldsymbol{z}$ & $\mathrm{U}(\mathbf{e q})$ \\
\hline U1 & $5958.8(2)$ & $4041.2(2)$ & 2500 & $11.01(9)$ \\
\hline Dy1 & 7500 & 7500 & $1676(16)$ & $10.5(6)$ \\
\hline S1 & 7500 & 2500 & 5000 & $8.2(3)$ \\
\hline S2 & $5407.1(8)$ & $6604.0(8)$ & $3838.3(13)$ & $10.80(16)$ \\
\hline Dy2 & 7500 & 7500 & $1050(20)$ & $10.4(8)$ \\
\hline
\end{tabular}


Table S11. Fractional Atomic Coordinates $\left(\times 10^{4}\right)$ and Equivalent Isotropic Displacement Parameters $\left(\AA^{2} \times 10^{3}\right)$ for $\mathrm{Ho}_{0.78(3)} \mathrm{U}_{2} \mathrm{~S}_{5}$. $\mathrm{U}_{\text {eq }}$ is defined as $1 / 3$ of the trace of the orthogonalised $\mathrm{U}_{\mathrm{IJ}}$ tensor.

\begin{tabular}{|c|c|c|c|c|}
\hline Atom & $\boldsymbol{x}$ & $\boldsymbol{y}$ & $\boldsymbol{z}$ & $\mathbf{U}(\mathbf{e q})$ \\
\hline U1 & $9037.2(2)$ & $4037.2(2)$ & 2500 & $11.29(16)$ \\
\hline Ho1 & 7500 & 7500 & $8320(20)$ & $12.6(10)$ \\
\hline S1 & 7500 & 2500 & 0 & $8.6(4)$ \\
\hline S2 & $8397.8(12)$ & $5408.4(12)$ & $6164.3(19)$ & $11.8(3)$ \\
\hline Ho2 & 7500 & 7500 & $9050(20)$ & $7.7(11)$ \\
\hline
\end{tabular}

Table S12. Fractional Atomic Coordinates $\left(\times 10^{4}\right)$ and Equivalent Isotropic Displacement Parameters $\left(\AA^{2} \times 10^{3}\right)$ for $\operatorname{Er}_{0.75(1)} \mathrm{U}_{2} \mathrm{~S}_{5}$. $\mathrm{U}_{\text {eq }}$ is defined as $1 / 3$ of the trace of the orthogonalised $\mathrm{U}_{\mathrm{IJ}}$ tensor.

\begin{tabular}{|c|c|c|c|c|}
\hline Atom & $\boldsymbol{x}$ & $\boldsymbol{y}$ & $\boldsymbol{z}$ & $\mathbf{U}(\mathbf{e q})$ \\
\hline U1 & $959.7(2)$ & $5959.7(2)$ & 2500 & $10.60(5)$ \\
\hline Er1 & 2500 & 2500 & $1716(8)$ & $9.4(3)$ \\
\hline S1 & $-408.1(6)$ & $6602.5(6)$ & $6158.7(9)$ & $10.70(10)$ \\
\hline S2 & 2500 & 7500 & 0 & $8.51(16)$ \\
\hline Er2 & 2500 & 2500 & $1042(9)$ & $10.4(4)$ \\
\hline
\end{tabular}

Table S13. Fractional Atomic Coordinates $\left(\times 10^{4}\right)$ and Equivalent Isotropic Displacement Parameters $\left(\AA^{2} \times 10^{3}\right)$ for $\mathrm{Tm}_{0.75(1)} \mathrm{U}_{2} \mathrm{~S}_{5}$. $\mathrm{U}_{\mathrm{eq}}$ is defined as $1 / 3$ of the trace of the orthogonalised $\mathrm{U}_{\mathrm{IJ}}$ tensor.

\begin{tabular}{|c|c|c|c|c|}
\hline Atom & $\boldsymbol{x}$ & $\boldsymbol{y}$ & $\boldsymbol{z}$ & $\mathbf{U}(\mathbf{e q})$ \\
\hline $\mathrm{U} 1$ & $960.1(2)$ & $5960.1(2)$ & 7500 & $9.47(6)$ \\
\hline $\mathrm{Tm} 1$ & 2500 & 2500 & $1731(8)$ & $8.3(4)$ \\
\hline $\mathrm{S} 1$ & 2500 & 7500 & 10000 & $7.59(19)$ \\
\hline $\mathrm{S} 2$ & $1602.3(6)$ & $4590.8(7)$ & $3842.7(10)$ & $9.80(11)$ \\
\hline $\mathrm{Tm} 2$ & 2500 & 2500 & $1038(9)$ & $9.4(4)$ \\
\hline
\end{tabular}


Table S14. Fractional Atomic Coordinates $\left(\times 10^{4}\right)$ and Equivalent Isotropic Displacement Parameters $\left(\AA^{2} \times 10^{3}\right)$ for $\mathrm{Yb}_{0.70(4)} \mathrm{U}_{2} \mathrm{~S}_{5}$. $\mathrm{U}_{\text {eq }}$ is defined as $1 / 3$ of the trace of the orthogonalised $\mathrm{U}_{\mathrm{IJ}}$ tensor.

\begin{tabular}{|c|c|c|c|c|}
\hline Atom & $\boldsymbol{x}$ & $\boldsymbol{y}$ & $\boldsymbol{z}$ & $\mathbf{U}(\mathbf{e q})$ \\
\hline $\mathrm{U} 1$ & $4043.3(2)$ & $5956.7(2)$ & 2500 & $10.5(3)$ \\
\hline $\mathrm{Yb} 1$ & 7500 & 7500 & $1720(30)$ & $9.2(10)$ \\
\hline $\mathrm{S} 1$ & $1602.9(13)$ & $4590.4(14)$ & $1152.6(19)$ & $10.5(3)$ \\
\hline $\mathrm{S} 2$ & 2500 & 7500 & 0 & $8.1(5)$ \\
\hline $\mathrm{Yb} 2$ & 7500 & 7500 & $1130(30)$ & $13.0(13)$ \\
\hline
\end{tabular}


Table S15. Fractional Atomic Coordinates $\left(\times 10^{4}\right)$ and Equivalent Isotropic Displacement Parameters $\left(\AA^{2} \times 10^{3}\right)$ for $\mathrm{Rb}_{1.72} \mathrm{Na}_{0.68} \mathrm{I}_{0.40}\left[\mathrm{Th}\left(\mathrm{PS}_{4}\right)_{2}\right]$. $\mathrm{U}_{\text {eq }}$ is defined as $1 / 3$ of the trace of the orthogonalised $\mathrm{U}_{\mathrm{IJ}}$ tensor.

\begin{tabular}{|c|c|c|c|c|}
\hline Atom & $\boldsymbol{x}$ & $\boldsymbol{y}$ & $\boldsymbol{z}$ & $\mathbf{U}(\mathbf{e q})$ \\
\hline $\mathrm{Th} 1$ & $3729.6(2)$ & $1593.8(2)$ & $1035.9(2)$ & $29.42(8)$ \\
\hline $\mathrm{Th} 2$ & 0 & 2500 & 2500 & $24.12(11)$ \\
\hline $\mathrm{I} 1$ & 7500 & 0 & $1639(3)$ & $100(5)$ \\
\hline $\mathrm{I} 2$ & $-1780(15)$ & $69(8)$ & $1975(3)$ & $133(7)$ \\
\hline $\mathrm{I} 3$ & $-1236(5)$ & $-357(2)$ & $2163.4(15)$ & $127(2)$ \\
\hline $\mathrm{Rb} 1$ & 2500 & 0 & $1984.0(7)$ & $119.7(14)$ \\
\hline $\mathrm{Rb} 2$ & $3190(40)$ & $197(13)$ & $2129(6)$ & $94(11)$ \\
\hline $\mathrm{Rb3}$ & 2500 & 0 & $-191.5(13)$ & $169(4)$ \\
\hline $\mathrm{Rb} 4$ & $716(11)$ & $-182(3)$ & $-79.8(19)$ & $146(4)$ \\
\hline $\mathrm{Rb} 5$ & 5000 & 2500 & $-201.3(5)$ & $107.6(9)$ \\
\hline $\mathrm{Rb} 6$ & $6235(4)$ & $-907.6(13)$ & $1516.0(14)$ & $58.3(8)$ \\
\hline $\mathrm{Rb} 7$ & -5000 & 2500 & 2500 & $95.5(13)$ \\
\hline $\mathrm{S} 1$ & $1317.0(19)$ & $1793.0(13)$ & $1343.3(5)$ & $46.1(5)$ \\
\hline $\mathrm{S} 2$ & $2930(2)$ & $2349.9(11)$ & $465.9(5)$ & $48.0(5)$ \\
\hline $\mathrm{S} 3$ & $6308.8(17)$ & $1965.3(9)$ & $1199.1(5)$ & $32.6(4)$ \\
\hline $\mathrm{S} 4$ & $3931.8(19)$ & $1648.5(11)$ & $1787.7(5)$ & $39.5(4)$ \\
\hline $\mathrm{S} 5$ & $4001(2)$ & $244.6(10)$ & $1204.0(7)$ & $48.8(5)$ \\
\hline $\mathrm{S} 6$ & $2129(2)$ & $793.5(9)$ & $596.5(5)$ & $39.0(4)$ \\
\hline $\mathrm{S} 7$ & $5523(2)$ & $1236.8(13)$ & $483.4(6)$ & $56.1(6)$ \\
\hline $\mathrm{S} 8$ & $8607(3)$ & $1317.9(18)$ & $694.1(9)$ & $75.4(9)$ \\
\hline $\mathrm{S} 9$ & $1101(2)$ & $1357.9(9)$ & $2178.9(6)$ & $40.3(5)$ \\
\hline $\mathrm{S} 10$ & $-1954(2)$ & $2171.8(9)$ & $2011.2(6)$ & $43.0(5)$ \\
\hline $\mathrm{P} 1$ & $2075.2(18)$ & $1907.4(9)$ & $1827.9(5)$ & $31.2(4)$ \\
\hline $\mathrm{P} 2$ & $6948.6(19)$ & $1762.8(10)$ & $697.6(5)$ & $36.7(4)$ \\
\hline $\mathrm{P} 3$ & 2500 & 0 & $894.7(7)$ & $32.9(5)$ \\
\hline $\mathrm{Na} 1$ & $6117(17)$ & $-1168(11)$ & $1495(5)$ & $115(8)$ \\
\hline $\mathrm{Na} 2$ & 7500 & 0 & $949(9)$ & $148(10)$ \\
\hline $\mathrm{Na3}$ & $-5810(40)$ & $3530(20)$ & $2402(8)$ & $102(12)$ \\
\hline & & & & \\
\hline
\end{tabular}


Table S16. Fractional Atomic Coordinates $\left(\times 10^{4}\right)$ and Equivalent Isotropic Displacement Parameters $\left(\AA^{2} \times 10^{3}\right)$ for $\mathrm{Cs}_{6} \mathrm{Cu}_{12} \mathrm{U}_{2} \mathrm{~S}_{15}$. $\mathrm{U}_{\mathrm{eq}}$ is defined as $1 / 3$ of the trace of the orthogonalised $\mathrm{U}_{\mathrm{IJ}}$ tensor.

\begin{tabular}{|c|c|c|c|c|}
\hline Atom & $\boldsymbol{x}$ & $\boldsymbol{y}$ & $\boldsymbol{z}$ & $\mathbf{U}(\mathbf{e q})$ \\
\hline $\mathrm{U} 1$ & 7500 & 2500 & 7500 & $14.95(13)$ \\
\hline $\mathrm{Cs} 1$ & $7834.4(2)$ & $5334.4(2)$ & 8750 & $26.30(15)$ \\
\hline $\mathrm{Cu} 1$ & $6733.9(4)$ & $3939.0(4)$ & $7585.3(5)$ & $24.8(2)$ \\
\hline $\mathrm{S} 1$ & 6250 & 5000 & 7500 & $19.2(6)$ \\
\hline S2 & $7841.7(8)$ & $3742.2(9)$ & $7943.0(9)$ & $20.9(3)$ \\
\hline
\end{tabular}




\section{Elemental Compositions}

Table S17. Elemental compositions of the $L n_{\mathrm{x}} \mathrm{U}_{2} \mathrm{~S}_{5}$ series and $\mathrm{Rb}_{1.72} \mathrm{Na}_{0.68} \mathrm{I}_{0.40}\left[\mathrm{Th}\left(\mathrm{PS}_{4}\right)_{2}\right]$ determined by EDS

\begin{tabular}{|c|c|c|c|c|c|c|c|}
\hline \multicolumn{2}{|c|}{$\mathrm{Pr}_{0.89(4)} \mathrm{U}_{2} \mathrm{~S}_{5}$} & \multicolumn{2}{c|}{$\mathrm{Nd}_{0.88(4)} \mathrm{U}_{2} \mathrm{~S}_{5}$} & \multicolumn{2}{c|}{$\mathrm{Sm}_{0.87(3)} \mathrm{U}_{2} \mathrm{~S}_{5}$} & \multicolumn{2}{c|}{$\mathrm{Gd}_{0.89(2)} \mathrm{U}_{2} \mathrm{~S}_{5}$} \\
\hline Element & Atom\% & Element & Atom\% & Element & Atom\% & Element & Atom\% \\
\hline $\mathrm{Pr}$ & 7.74 & $\mathrm{Nd}$ & 7.00 & $\mathrm{Sm}$ & 9.97 & $\mathrm{Gd}$ & 9.12 \\
\hline $\mathrm{U}$ & 40.66 & $\mathrm{U}$ & 30.78 & $\mathrm{U}$ & 39.20 & $\mathrm{U}$ & 24.20 \\
\hline $\mathrm{S}$ & 51.60 & $\mathrm{~S}$ & 62.22 & $\mathrm{~S}$ & 50.83 & $\mathrm{~S}$ & 66.68 \\
\hline
\end{tabular}

\begin{tabular}{|c|c|c|c|c|c|c|c|}
\hline \multicolumn{2}{|c|}{$\mathrm{Tb}_{0.82(3)} \mathrm{U}_{2} \mathrm{~S}_{5}$} & \multicolumn{2}{c|}{$\mathrm{Dy}_{0.76(3)} \mathrm{U}_{2} \mathrm{~S}_{5}$} & \multicolumn{2}{c|}{$\mathrm{Ho}_{0.79(3)} \mathrm{U}_{2} \mathrm{~S}_{5}$} & \multicolumn{2}{c|}{$\mathrm{Er}_{0.75(1)} \mathrm{U}_{2} \mathrm{~S}_{5}$} \\
\hline Element & Atom\% & Element & Atom\% & Element & Atom\% & Element & Atom\% \\
\hline $\mathrm{Tb}$ & 11.64 & $\mathrm{Dy}$ & 9.89 & $\mathrm{Ho}$ & 9.09 & $\mathrm{Er}$ & 8.71 \\
\hline $\mathrm{U}$ & 36.28 & $\mathrm{U}$ & 41.40 & $\mathrm{U}$ & 25.69 & $\mathrm{U}$ & 43.97 \\
\hline $\mathrm{S}$ & 52.08 & $\mathrm{~S}$ & 48.71 & $\mathrm{~S}$ & 65.22 & $\mathrm{~S}$ & 47.32 \\
\hline
\end{tabular}

\begin{tabular}{|c|c|c|c|c|c|}
\hline \multicolumn{2}{|c|}{$\mathrm{Tm}_{0.75(1)} \mathrm{U}_{2} \mathrm{~S}_{5}$} & \multicolumn{2}{|c|}{$\mathrm{Yb}_{0.70(4)} \mathrm{U}_{2} \mathrm{~S}_{5}$} & \multicolumn{2}{|c|}{$\mathrm{Rb}_{1.72} \mathrm{Na}_{0.68} \mathrm{I}_{0.40}\left[\mathrm{Th}\left(\mathrm{PS}_{4}\right)_{2}\right]$} \\
\hline Element & Atom $\%$ & Element & Atom $\%$ & Element & Atom $\%$ \\
\hline $\mathrm{Tm}$ & 7.82 & $\mathrm{Yb}$ & 6.19 & $\mathrm{Rb}$ & 13.09 \\
\hline $\mathrm{U}$ & 25.72 & $\mathrm{U}$ & 30.51 & $\mathrm{Na}$ & 8.83 \\
\hline $\mathrm{S}$ & 66.46 & $\mathrm{~S}$ & 63.30 & $\mathrm{I}$ & 3.84 \\
\hline & & & & $\mathrm{Th}$ & 9.66 \\
\hline & & & & $\mathrm{P}$ & 21.09 \\
\hline & & & & $\mathrm{S}$ & 43.50 \\
\hline
\end{tabular}


13. SEM images

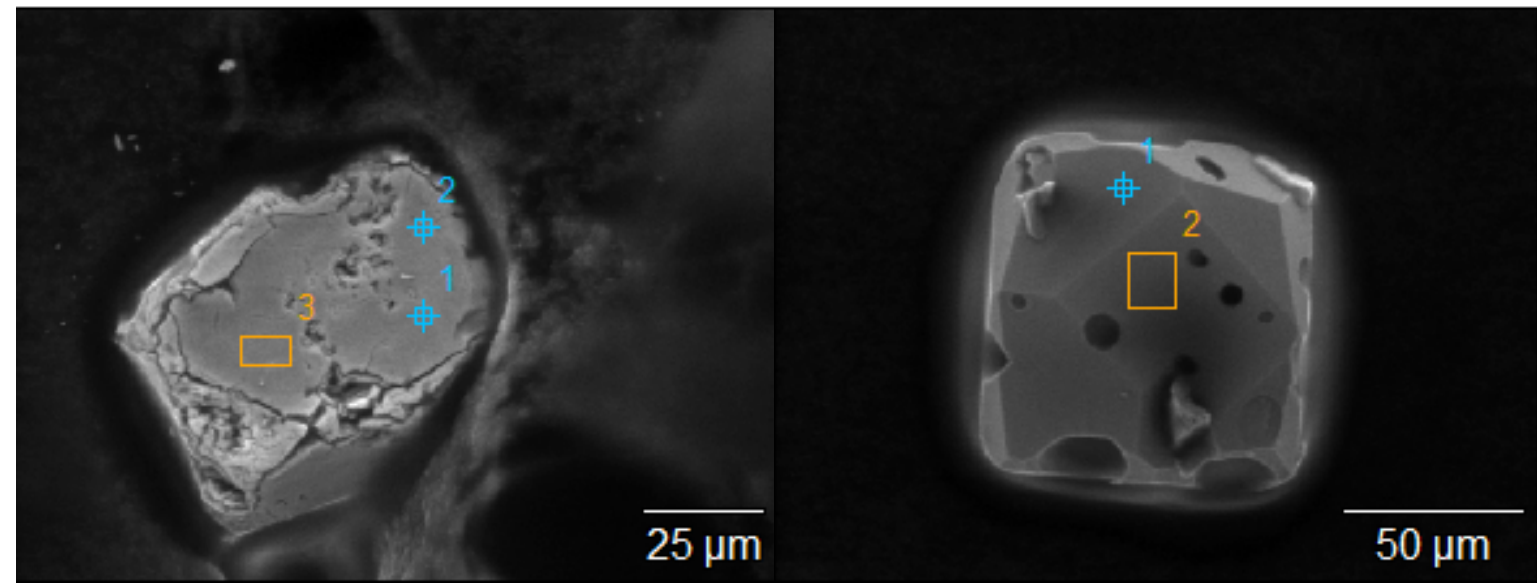

(a)

(b)

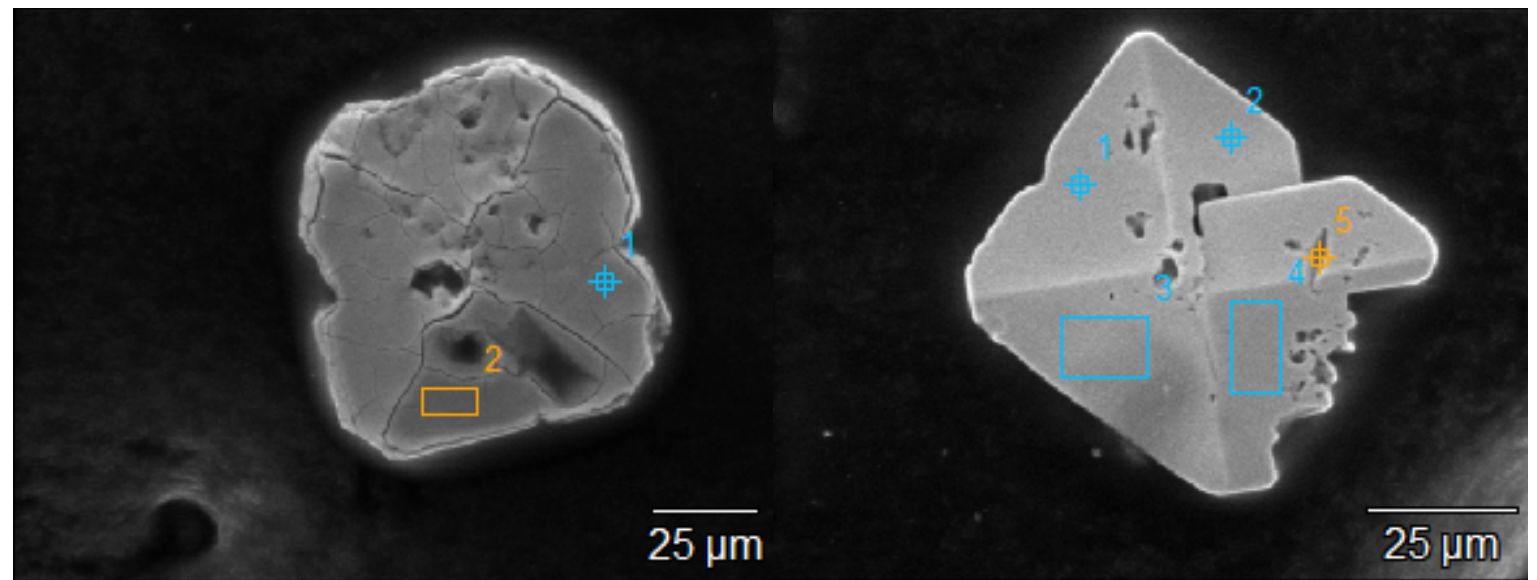

(c)

(d)

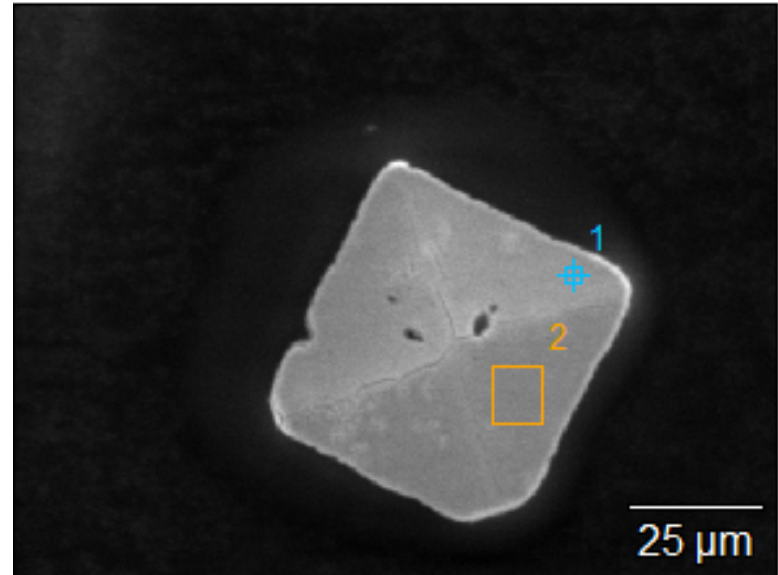

$25 \mu \mathrm{m}$

(e)

(f) 


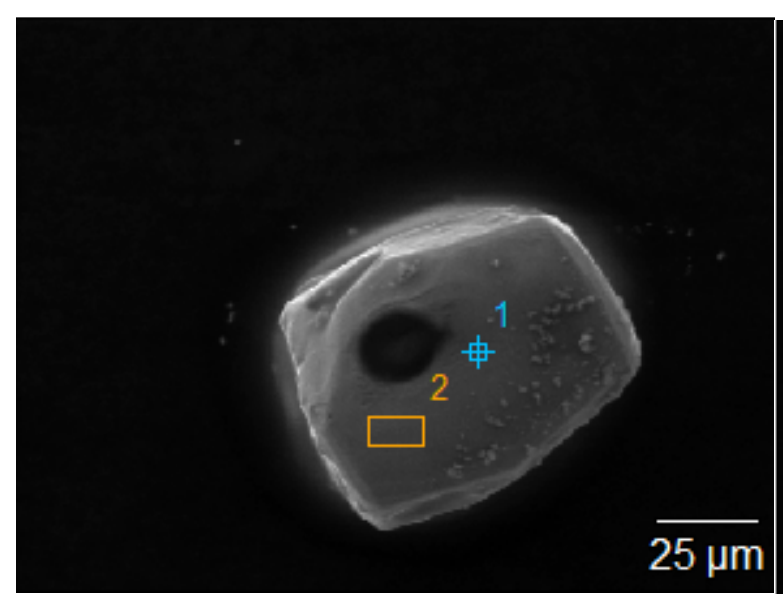

$(\mathrm{g})$

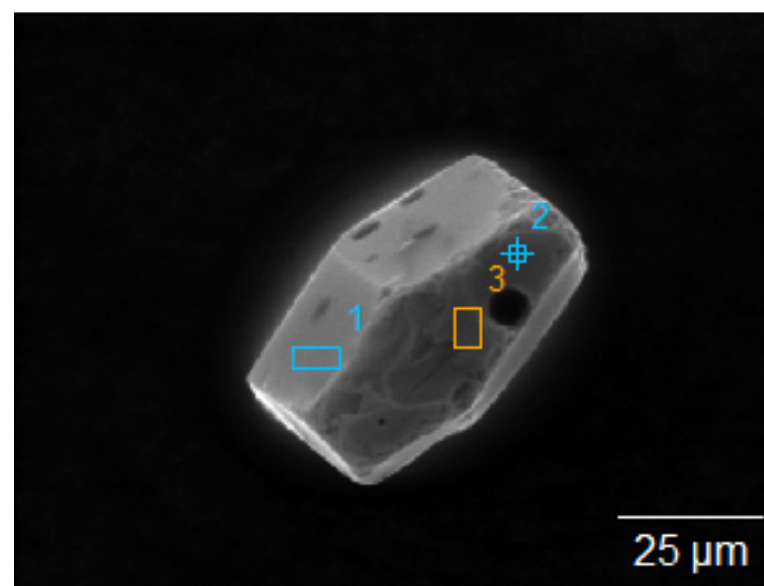

(i)

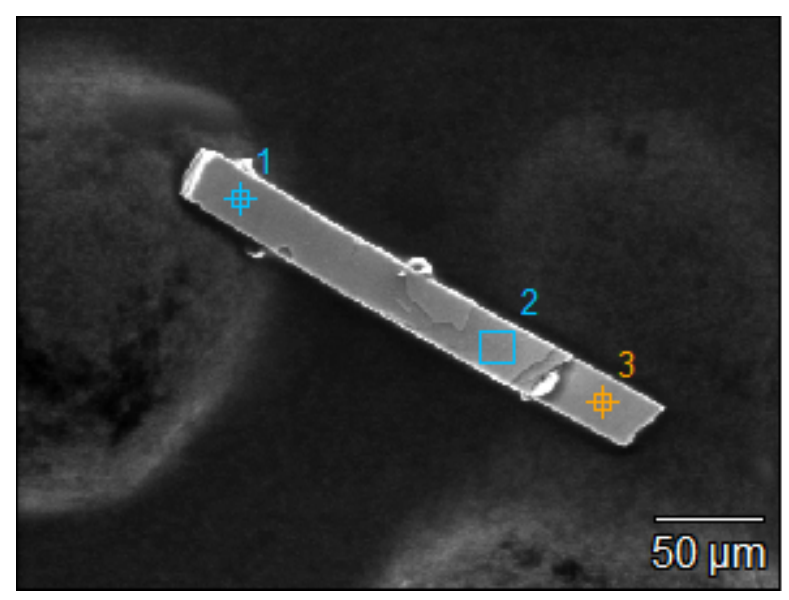

(k)
$25 \mu \mathrm{m}$

(h)

$50 \mu \mathrm{m}$

(j)

Figure S22. SEM images of single crystal of (a) $\operatorname{Pr}_{0.89(4)} \mathrm{U}_{2} \mathrm{~S}_{5}$, (b) $\mathrm{Nd}_{0.88(4)} \mathrm{U}_{2} \mathrm{~S}_{5}$, (c) $\mathrm{Sm}_{0.87(3)} \mathrm{U}_{2} \mathrm{~S}_{5}$, (d) $\mathrm{Gd}_{0.89(2)} \mathrm{U}_{2} \mathrm{~S}_{5}$, (e) $\mathrm{Tb}_{0.82(3)} \mathrm{U}_{2} \mathrm{~S}_{5}$, (f) $\mathrm{Dy}_{0.76(3)} \mathrm{U}_{2} \mathrm{~S}_{5}$, (g) $\mathrm{Ho}_{0.79(3)} \mathrm{U}_{2} \mathrm{~S}_{5}$, (h) $\mathrm{Er}_{0.75(1)} \mathrm{U}_{2} \mathrm{~S}_{5}$, (i) $\mathrm{Tm}_{0.75(1)} \mathrm{U}_{2} \mathrm{~S}_{5},(\mathrm{j}) \mathrm{Yb}_{0.70(4)} \mathrm{U}_{2} \mathrm{~S}_{5}$, and (k) $\mathrm{Rb}_{1.72} \mathrm{Na}_{0.68} \mathrm{I}_{0.40}\left[\mathrm{Th}_{(}\left(\mathrm{PS}_{4}\right)_{2}\right]$. 


\section{SEM Spectra}

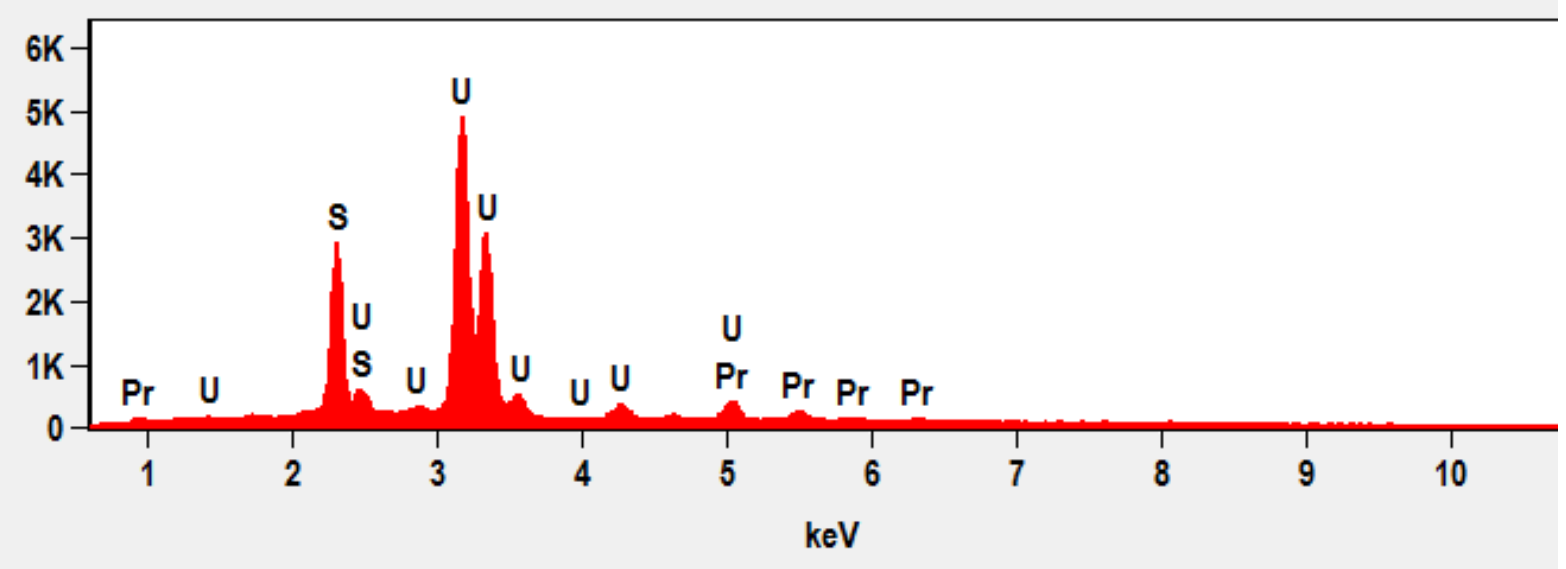

Figure S23. EDS spectrum of $\operatorname{Pr}_{0.89(4)} \mathrm{U}_{2} \mathrm{~S}_{5}$.

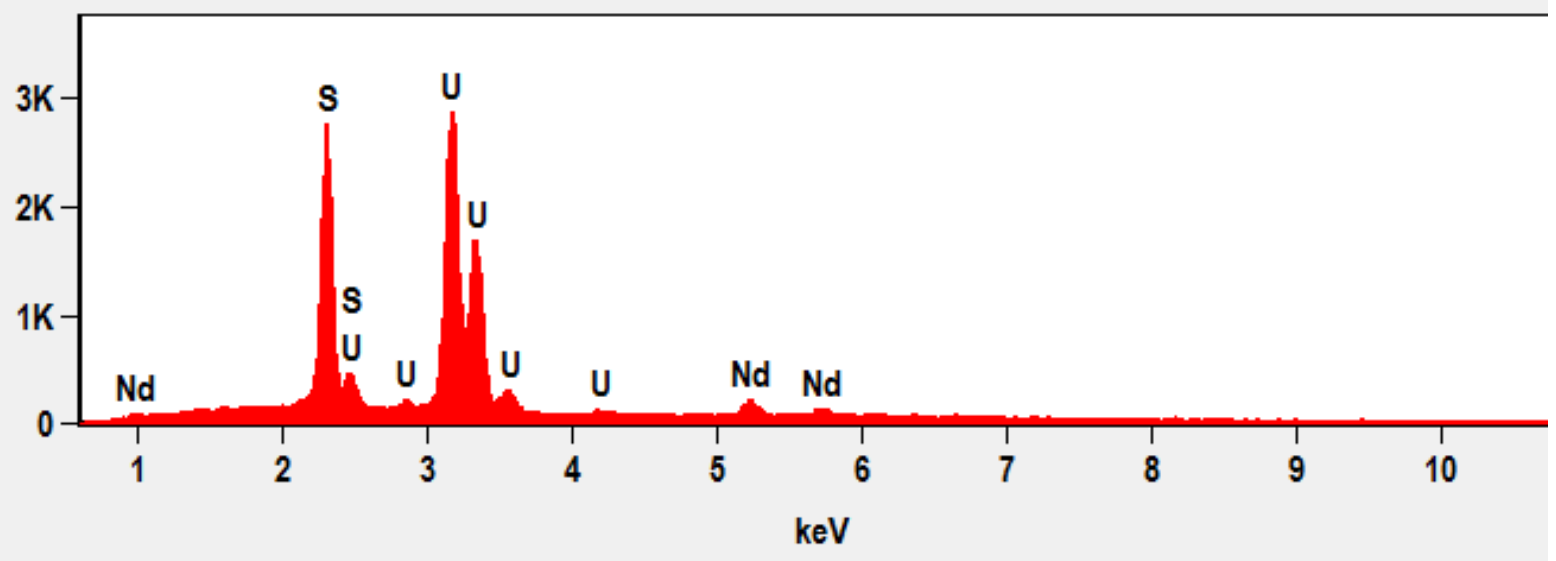

Figure S24. EDS spectrum of $\mathrm{Nd}_{0.88(4)} \mathrm{U}_{2} \mathrm{~S}_{5}$. 


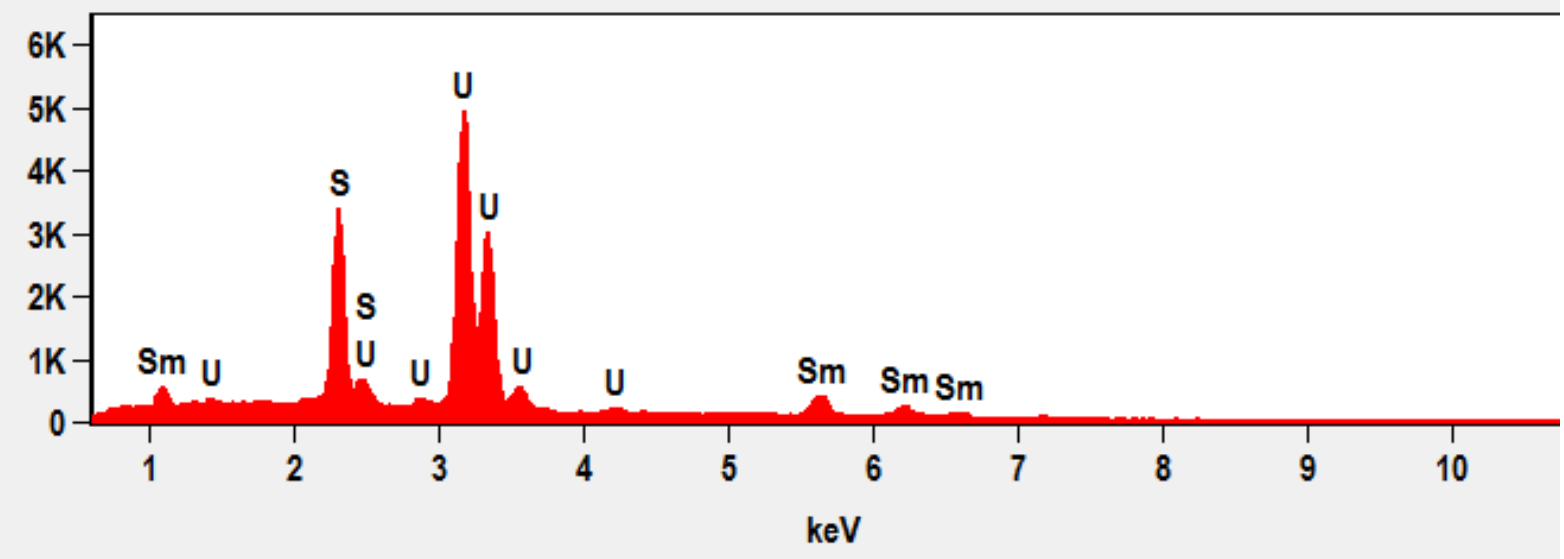

Figure $\mathrm{S} 25$. EDS spectrum of $\mathrm{Sm}_{0.87(3)} \mathrm{U}_{2} \mathrm{~S}_{5}$.

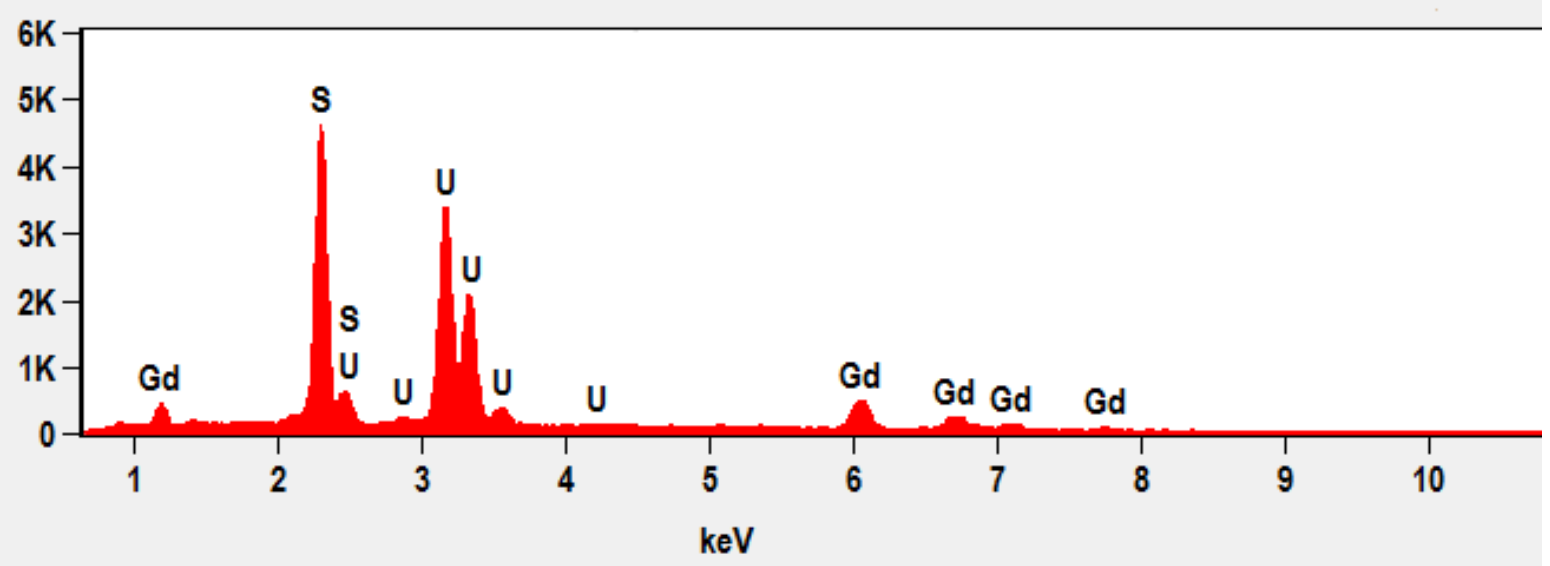

Figure $\mathrm{S} 26$. EDS spectrum of $\mathrm{Gd}_{0.88(2)} \mathrm{U}_{2} \mathrm{~S}_{5}$. 


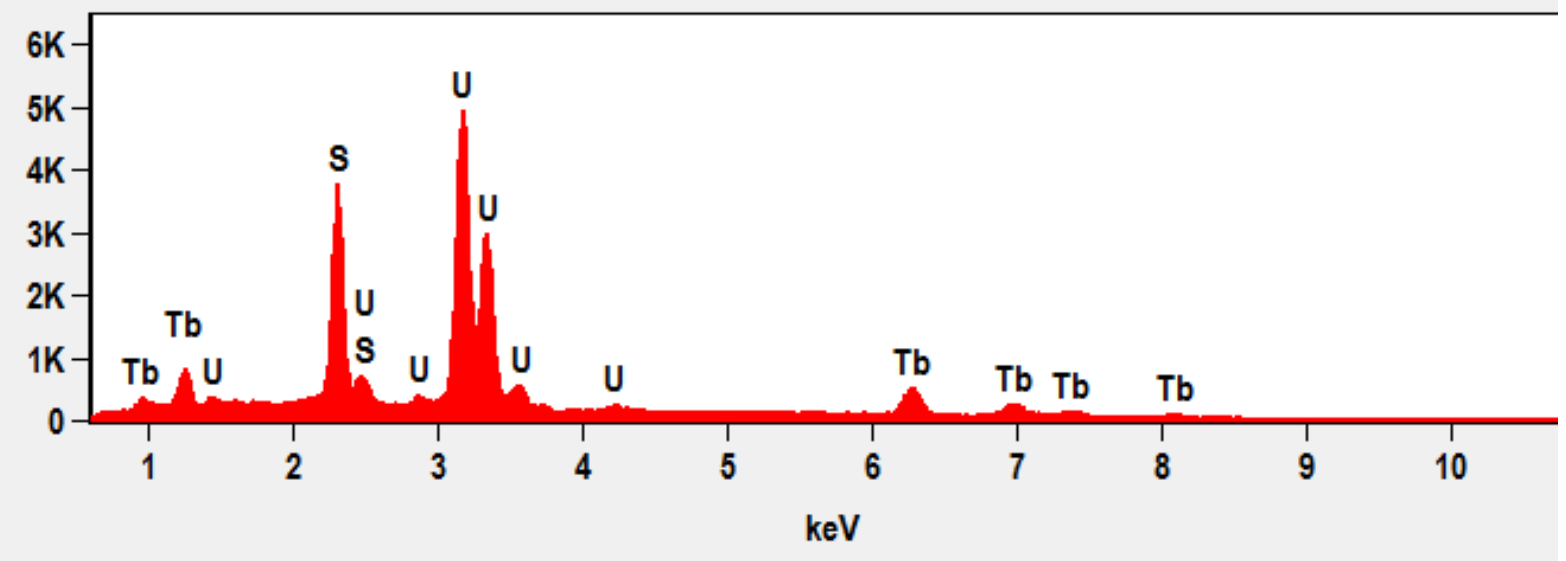

Figure S27. EDS spectrum of $\mathrm{Tb}_{0.82(3)} \mathrm{U}_{2} \mathrm{~S}_{5}$.

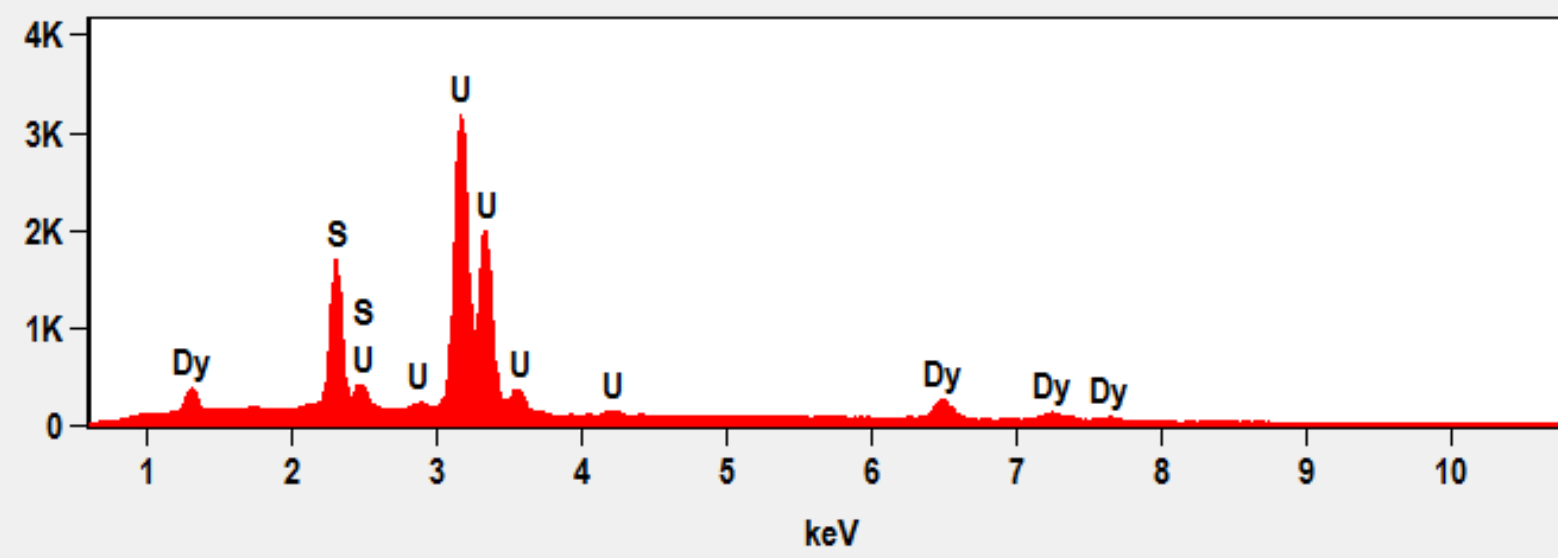

Figure S28. EDS spectrum of $\mathrm{Dy}_{0.76(3)} \mathrm{U}_{2} \mathrm{~S}_{5}$. 


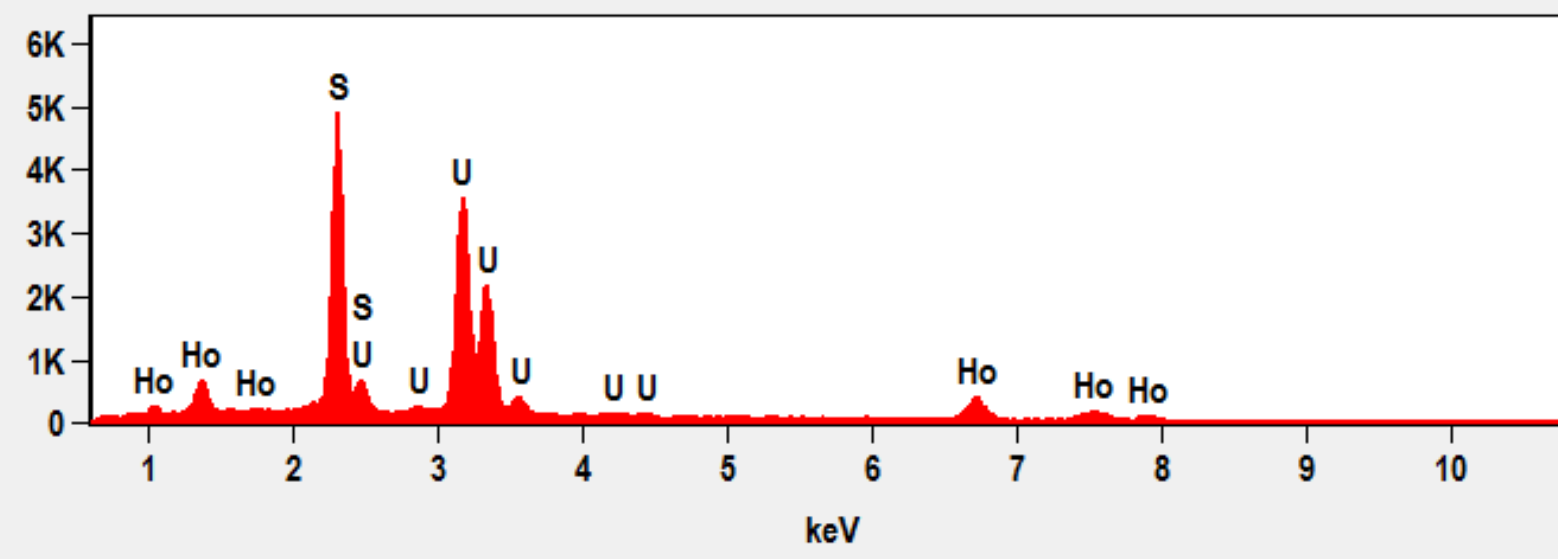

Figure S29. EDS spectrum of $\mathrm{Ho}_{0.78(3)} \mathrm{U}_{2} \mathrm{~S}_{5}$.

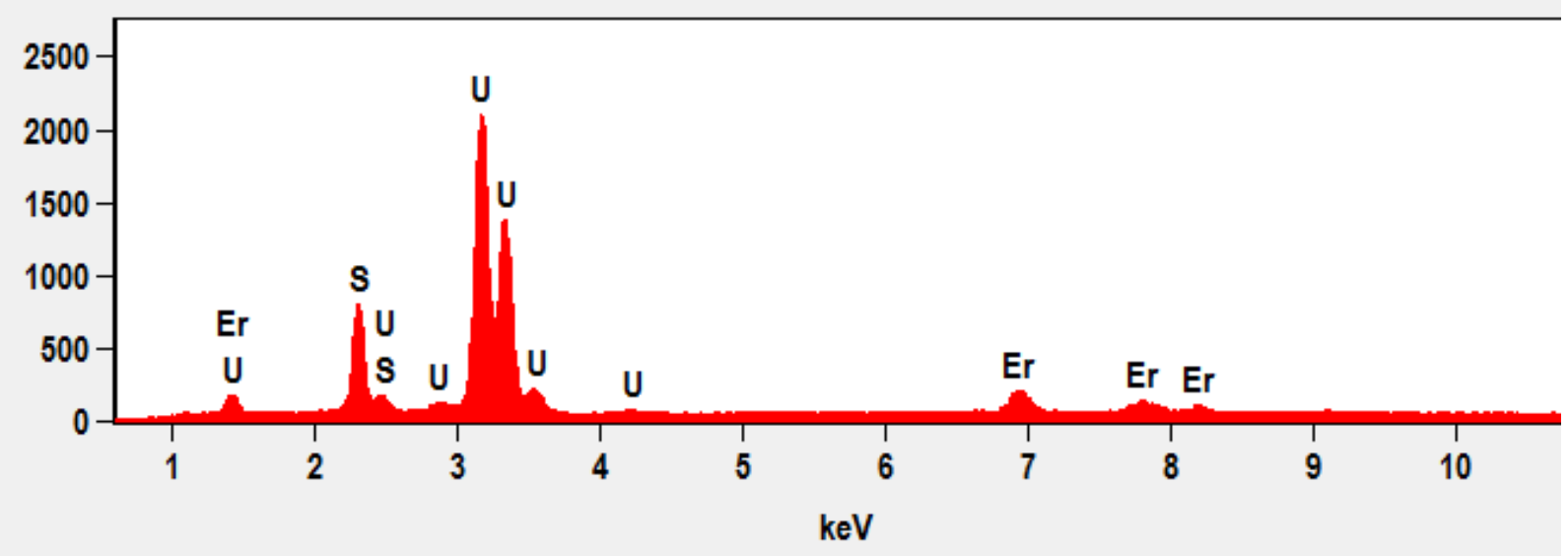

Figure S30. EDS spectrum of $\operatorname{Er}_{0.75(1)} \mathrm{U}_{2} \mathrm{~S}_{5}$. 


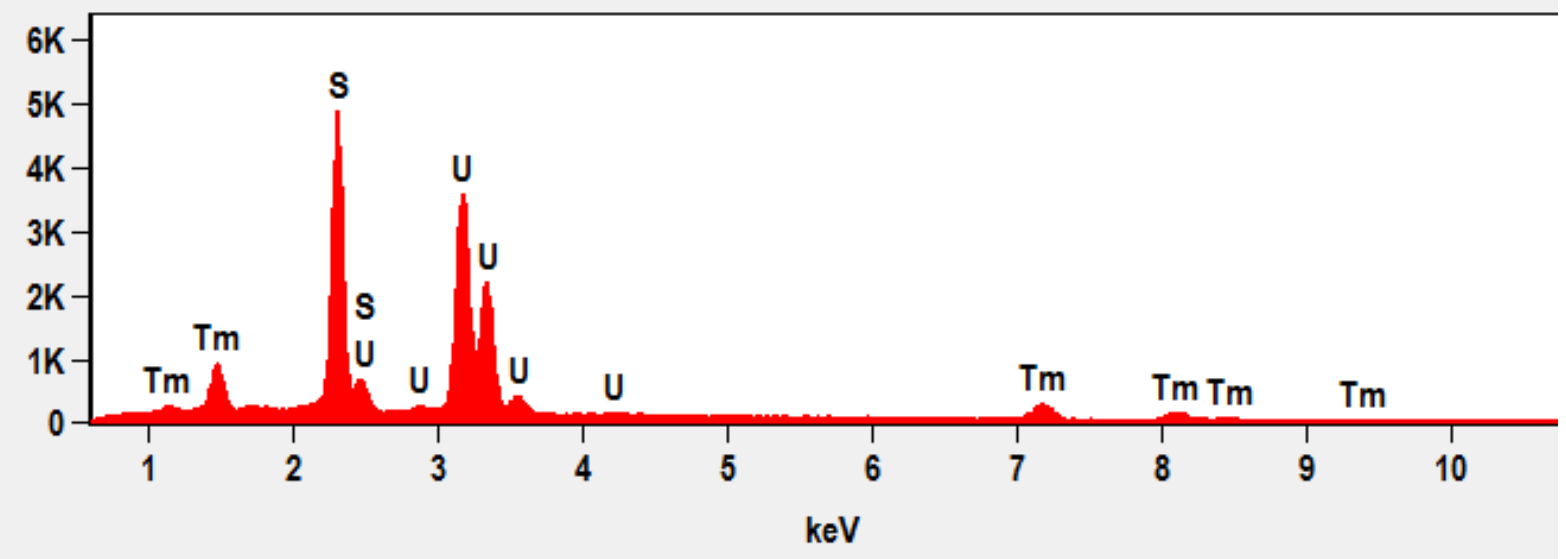

Figure S31. EDS spectrum of $\mathrm{Tm}_{0.75(1)} \mathrm{U}_{2} \mathrm{~S}_{5}$.

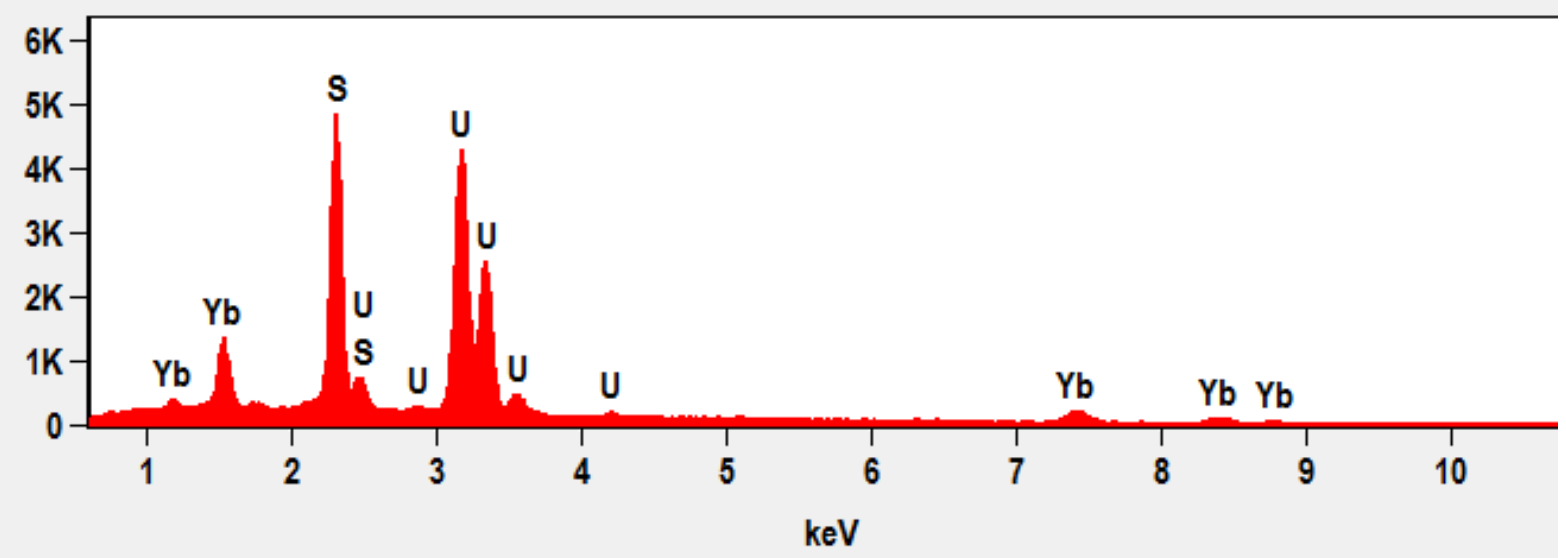

Figure $\mathrm{S} 32$. EDS spectrum of $\mathrm{Yb}_{0.70(4)} \mathrm{U}_{2} \mathrm{~S}_{5}$. 


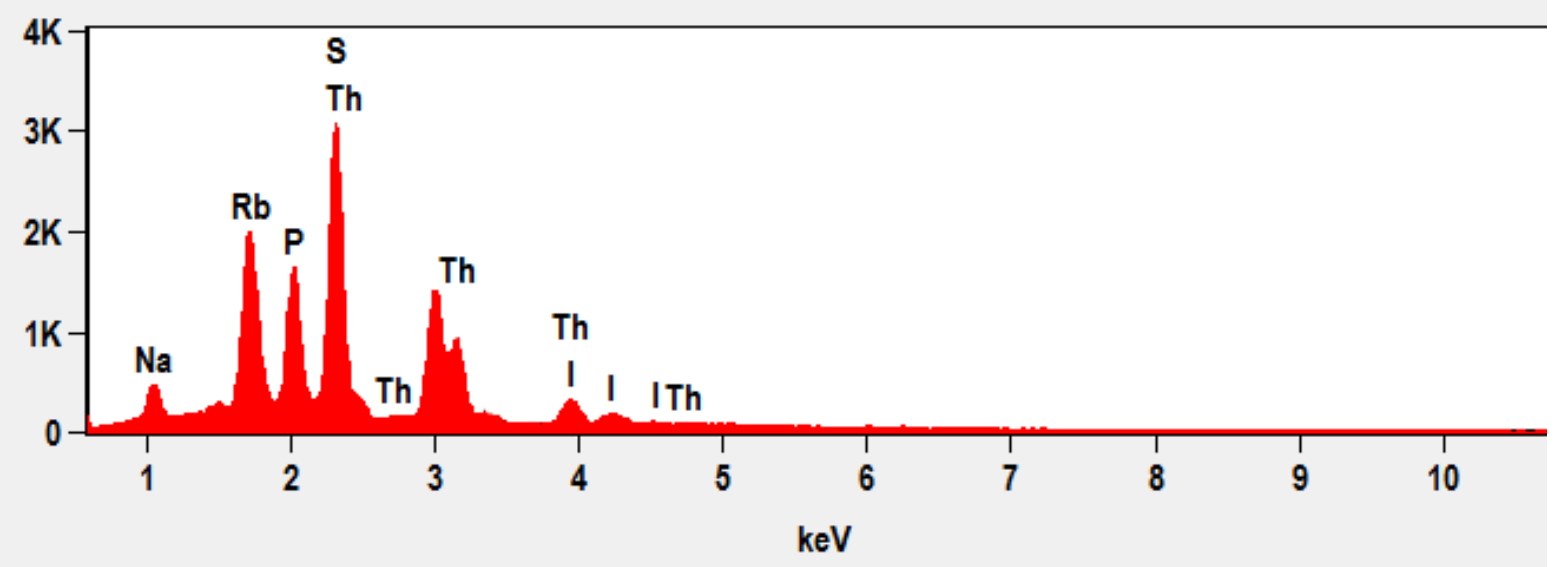

Figure S33. EDS spectrum for $\mathrm{Rb}_{1.72} \mathrm{Na}_{0.68} \mathrm{I}_{0.40}\left[\mathrm{Th}\left(\mathrm{PS}_{4}\right)_{2}\right]$. 
15. Tabulated Reagent Ratios and Amounts for the Chalcogenation Reactions

Table S18. The molar ratios and amounts of reagents used in the chalcogenation reactions all at a reaction temperature of $800{ }^{\circ} \mathrm{C}$

\begin{tabular}{|c|c|c|c|c|c|}
\hline $\begin{array}{l}\text { Actinide } \\
\text { Source }\end{array}$ & Chalcogen & $\begin{array}{c}\text { Molar Ratio } \\
\text { An:B:S }\end{array}$ & $\begin{array}{c}\text { mmols Actinide } \\
\text { Oxide }\end{array}$ & $\begin{array}{c}\text { mmols } \\
\text { Boron } \\
\end{array}$ & $\begin{array}{l}\text { mmols } \\
\text { Sulfur }\end{array}$ \\
\hline \multirow{6}{*}{$\mathrm{UO}_{2}$} & \multirow{2}{*}{$\mathrm{S}$} & $1: 2: 3$ & 0.741 & 1.48 & 2.224 \\
\hline & & $1: 4: 6$ & 0.37 & 1.48 & 2.224 \\
\hline & \multirow{2}{*}{$\mathrm{Se}$} & $1: 2: 3$ & 0.741 & 1.48 & 2.224 \\
\hline & & $1: 4: 6$ & 0.741 & 2.96 & 4.444 \\
\hline & \multirow{2}{*}{$\mathrm{Te}$} & $1: 2: 3$ & 0.37 & 0.74 & 1.111 \\
\hline & & $1: 4: 6$ & 0.37 & 1.48 & 2.223 \\
\hline \multirow{6}{*}{$\mathrm{ThO}_{2}$} & \multirow{2}{*}{$\mathrm{S}$} & $1: 2: 3$ & 0.379 & 0.758 & 1.135 \\
\hline & & $1: 4: 6$ & 0.379 & 1.517 & 2.272 \\
\hline & \multirow{2}{*}{$\mathrm{Se}$} & $1: 2: 3$ & 0.757 & 1.517 & 2.272 \\
\hline & & $1: 4: 6$ & 0.757 & 3.034 & 4.545 \\
\hline & \multirow{2}{*}{$\mathrm{Te}$} & $1: 2: 3$ & 0.379 & 0.758 & 1.137 \\
\hline & & $1: 4: 6$ & 0.379 & 1.517 & 2.275 \\
\hline
\end{tabular}

Table S19. The molar ratios and amounts of reagents used in the sulfurization reactions at temperatures of $400-800{ }^{\circ} \mathrm{C}$ and $500-800{ }^{\circ} \mathrm{C}$ for reactions involving uranium and thorium, respectively

\begin{tabular}{|c|c|c|c|c|c|}
\hline $\begin{array}{c}\text { Actinide } \\
\text { Source }\end{array}$ & Chalcogen & $\begin{array}{c}\text { molar ratio } \\
\text { An:B:S }\end{array}$ & $\begin{array}{c}\text { mmols Actinide } \\
\text { Oxide }\end{array}$ & $\begin{array}{c}\text { mmols } \\
\text { Boron }\end{array}$ & $\begin{array}{c}\text { mmols } \\
\text { Sulfur }\end{array}$ \\
\hline $\mathrm{U}_{3} \mathrm{O}_{8}$ & $\mathrm{~S}$ & $1: 6: 9$ & 0.237 & 1.424 & 2.136 \\
\hline $\mathrm{ThO}_{2}$ & $\mathrm{~S}$ & $1: 2: 3$ & 0.189 & 0.378 & 0.567 \\
\hline
\end{tabular}


16. $L n_{\mathrm{x}} \mathrm{U}_{2} \mathrm{~S}_{5}$ Structure Figure
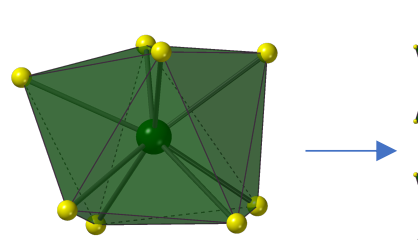

$\mathrm{US}_{8}$ distorted bicapped trigonal prism
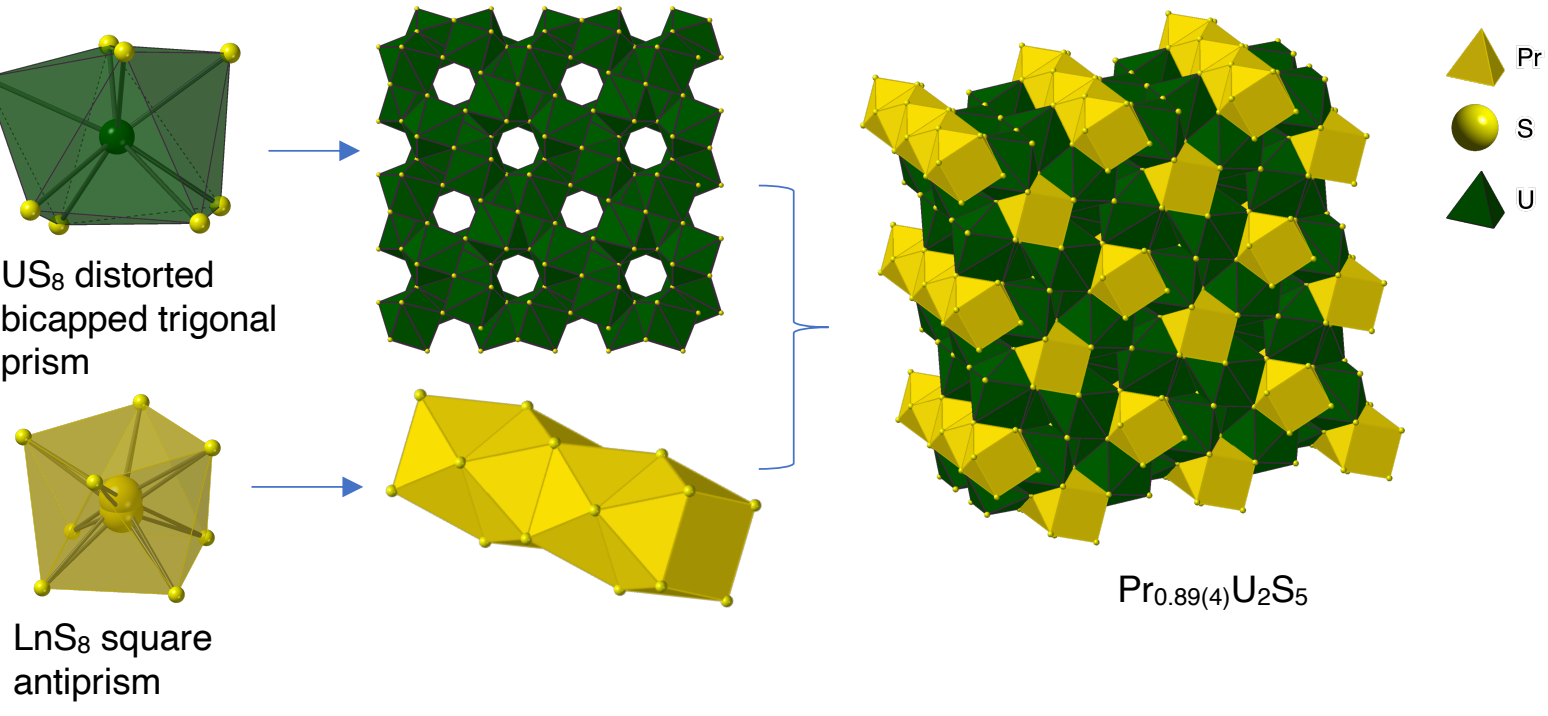

Figure S34. A view on the structure of $\operatorname{Pr}_{0.89(4)} \mathrm{U}_{2} \mathrm{~S}_{5}$ series. $\mathrm{US}_{8}$ distorted bicapped trigonal prisms edge share to create a 3-dimensional structure with voids that run through which are filled with face sharing $\mathrm{LnS}_{8}$ square antiprism. The lanthanides are disordered within their polyhedra. 


\section{BVS Results}

Table S20. Bond Valence Sums (BVS) were performed on the $L n_{\mathrm{x}} \mathrm{U}_{2} \mathrm{~S}_{5}$ series. The U-S distances are tabulated below along with the calculated uranium oxidation state. Each bond length describes two of the bonds in the 8-coordinate uranium polyhedra causing each to be counted twice in the BVS calculation. The calculations suggest an average uranium valency of 3.76 further supporting the assignment of mixed $\mathrm{U}^{3+} / \mathrm{U}^{4+}$ in these structures.

\begin{tabular}{|c|c|c|c|c|c|}
\hline Composition & \multicolumn{4}{|c|}{$\mathrm{U}-\mathrm{S}$ bond distances } & Calculated U oxidation state \\
\hline $\mathrm{Pr}_{0.89(4)} \mathrm{U}_{2} \mathrm{~S}_{5}$ & 2.83 & 2.80 & 3.00 & 2.75 & 3.70 \\
\hline $\mathrm{Nd}_{0.88(4)} \mathrm{U}_{2} \mathrm{~S}_{5}$ & 2.80 & 2.83 & 2.99 & 2.75 & 3.72 \\
\hline $\mathrm{Sm}_{0.87(3)} \mathrm{U}_{2} \mathrm{~S}_{5}$ & 2.83 & 2.80 & 2.99 & 2.74 & 3.73 \\
\hline $\mathrm{Gd}_{0.89(2)} \mathrm{U}_{2} \mathrm{~S}_{5}$ & 2.74 & 2.84 & 2.99 & 2.81 & 3.72 \\
\hline $\mathrm{Tb}_{0.82(3)} \mathrm{U}_{2} \mathrm{~S}_{5}$ & 2.74 & 2.80 & 2.99 & 2.83 & 3.78 \\
\hline $\mathrm{Dy}_{0.76(3)} \mathrm{U}_{2} \mathrm{~S}_{5}$ & 2.74 & 2.79 & 2.99 & 2.82 & 3.83 \\
\hline $\mathrm{Ho}_{0.79(3)} \mathrm{U}_{2} \mathrm{~S}_{5}$ & 2.73 & 2.79 & 2.99 & 2.82 & 3.86 \\
\hline $\mathrm{Er}_{0.75(1)} \mathrm{U}_{2} \mathrm{~S}_{5}$ & 2.99 & 2.78 & 2.99 & 2.74 & 3.50 \\
\hline $\mathrm{Tm}_{0.75(1)} \mathrm{U}_{2} \mathrm{~S}_{5}$ & 2.73 & 2.78 & 2.99 & 2.82 & 3.87 \\
\hline $\mathrm{Yb}_{0.70(4)} \mathrm{U}_{2} \mathrm{~S}_{5}$ & 2.99 & 2.78 & 2.81 & 2.74 & 3.89 \\
\hline
\end{tabular}

